\title{
Exploiting the Reuse Supplied by Loop-Dependent Stream References for Stream Processors
}

\author{
XUEJUN YANG, YING ZHANG, and XICHENG LU \\ National University of Defense Technology \\ JINGLING XUE \\ The University of New South Wales \\ IAN ROGERS \\ The University of Manchester \\ and \\ GEN LI, GUIBIN WANG, and XUDONG FANG \\ National University of Defense Technology
}

Memory accesses limit the performance of stream processors. By exploiting the reuse of data
held in the Stream Register File (SRF), an on-chip, software controlled storage, the number of
memory accesses can be reduced. In current stream compilers, reuse exploitation is only at-
tempted for simple stream references, those whose start and end are known. Compiler analy-
sis, from outside of stream processors, does not directly enable the consideration of other more
complex stream references. In this article, we propose a transformation to automatically opti-
mize stream programs to exploit the reuse supplied by loop-dependent stream references. The
transformation is based on three results: lemmas identifying the reuse supplied by stream

A preliminary version of this article entitled Exploiting Loop-Dependent Stream Reuse for Stream Processors appeared in Proceedings of the 17th International Conference on Parallel Architectures and Compilation Techniques [Yang et al. 2008]. This extended version makes the following new contributions over the previous paper: (i) it analyzes the SRF pressure brought by the stream reuse exploration; (ii) it selects the streams to reuse such that as many memory accesses are eliminated as possible within the limited SRF capacity by modeling the task as a knapsack problem; (iii) it uses a greedy approximation algorithm to find a solution, which is independent of stream processor architecture, to improve SRF utilization; (iv) it changes the unrolling factor of the loop from the least common multiple of all sub-RGs' unrolling factors to the maximum of them.

This work was supported by NSFC (61003075, 60921062, 60633050).

Author's addresses: X. Yang, Y. Zhang, X. Lu, National University of Defense Technology, China; email: zhangying@nudt.edu.cn; J. Xue, The University of New South Wales, Sydney, Australia; I. Rogers, The University of Manchester, Manchester, UK; G. Li, G. Wang, and X. Fang, School of Computer, National University of Defense Technology, Changsha, China.

Permission to make digital or hard copies of part or all of this work for personal or classroom use is granted without fee provided that copies are not made or distributed for profit or commercial advantage and that copies show this notice on the first page or initial screen of a display along with the full citation. Copyrights for components of this work owned by others than ACM must be honored. Abstracting with credit is permitted. To copy otherwise, to republish, to post on servers, to redistribute to lists, or to use any component of this work in other works requires prior specific permission and/or a fee. Permissions may be requested from Publications Dept., ACM, Inc., 2 Penn Plaza, Suite 701, New York, NY 10121-0701 USA, fax +1 (212) 869-0481, or permissions@acm.org. (C) 2010 ACM 1544-3566/2010/09-ART11 $\$ 10.00$

DOI 10.1145/1839667.1839673 http://doi.acm.org/10.1145/1839667.1839673

ACM Transactions on Architecture and Code Optimization, Vol. 7, No. 2, Article 11, Pub. date: September 2010. 
references, a new abstract representation called the Stream Reuse Graph (SRG) depicting the identified reuse, and the optimization of the SRG for our transformation. Both the reuse between the whole sequences accessed by stream references and between partial sequences is exploited in the article. In particular, partial reuse and its treatment are quite new and have never, to the best of our knowledge, appeared in scalar and vector processing. At the same time, reusing streams increases the pressure on the SRF, and this presents a problem of which reuse should be exploited within limited SRF capacity. We extend our analysis to achieve this objective. Finally, we implement our techniques based on the StreamC/KernelC compiler that has been optimized with the best existing compilation techniques for stream processors. Experimental results show a resultant speed-up of 1.14 to 2.54 times using a range of benchmarks.

Categories and Subject Descriptors: D.3.4 [Programming Languages]: Processors, Compilers; D.3.2 [Programming Languages]: Language Classifications, Specialized Application Languages

General Terms: Algorithms, Design, Management, Performance, Experimentation

Additional Key Words and Phrases: Stream programming model, stream professor, stream register file, streamc, stream reuse

ACM Reference Format:

Yang, X., Zhang, Y., Lu, X., Xue, J., Rogers, I., Li, G., Wang, G., and Fang, X. 2010. Exploiting the reuse supplied by loop-dependent stream references for stream processors. ACM Trans. Architec. Code Optim. 7, 2, Article 11 (September 2010), 35 pages.

DOI = 10.1145/1839667.1839673 http://doi.acm.org/10.1145/1839667.1839673.

\section{INTRODUCTION}

In the past, with increasing clock frequencies, and now, with an increasing number of cores, processing power is doubling every 18 months [Agarwal et al. 2000; Horowitz and Dally 2004]. Memory access time has also been increasing but at a slower rate, leading to what has become to be known as the memory wall [Wulf and McKee 1995]. Architectural and software techniques, including reusing data on-chip and prefetching to hide long memory latency, have been applied to relieve the memory wall. The research into advanced processors now focuses on two areas: developing multicore as well as multithreaded general-purpose processors, and developing stream processors using novel architectural models, such as the Imagine stream processor [Kapasi et al. 2002], the Merrimac stream processor [Dally et al. 2003], the Cell processor [Kahle et al. 2005], the FT64 stream processor [Yang et al. 2007], RAW [Taylor et al. 2002] and GPUs (e.g., AMD FireStream [AMD Inc.], NVIDIA Tesla [Lindholm et al. 2008] and Intel Larrabee [Seiler et al. 2008]). Much research has been put into reusing on-chip data for a conventional architectural model [Allen and Kennedy 1992; Callahan et al. 1990; Lu and Cooper 1997; Carr and Kennedy 1994; Sastry and Ju 1998; Lo et al. 1998; Xue and Huang 1997]; however, reusing on-chip data for a stream processor is a less mature research area.

Stream processors were originally developed for media applications [Owens et al. 2002] that have little to no data reuse, especially the reuse across different loops [Crow 2004]. However, stream processors have now been used for scientific applications [Dally et al. 2003; Yang et al. 2007] that have much more complicated and irregular dependence and reuse. Therefore, exploiting stream reuse within programs is now an important and necessary work. 

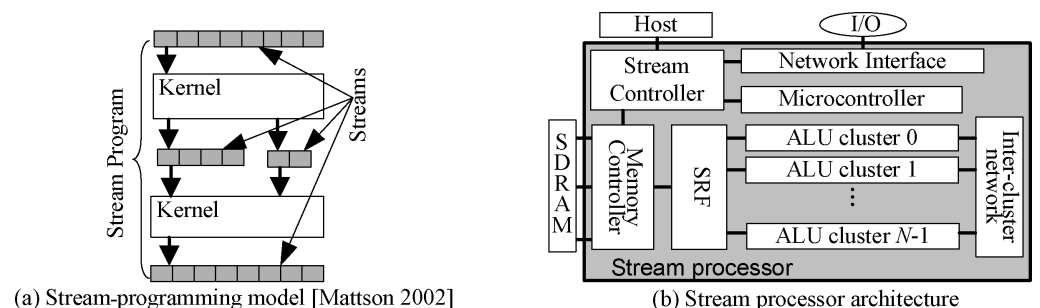

Fig. 1. Stream processing.

\subsection{Stream Processing}

The stream-programming model [Mattson 2002; Dally et al. 2003; Owens et al. 2002; Stephens 1997; Kapasi et al. 2003; Kapasi et al. 2002; Yang et al. 2007; Buck et al. 2004] shown in Figure 1(a), divides an application into a stream-level program and one or more kernels that define each processing step. The stream-level program declares the streams and defines the high-level control and data flow between kernels. Each kernel is a function that consumes and produces elements from streams. A stream is a sequence of data records.

Many research groups have developed architectures for stream applications. The stream architecture design space spans from configurable or statically scheduled tiled processors (Raw [Taylor et al. 2002]), to SIMD stream coprocessors with a large local memory for stream buffering (the Imagine stream processor [Kapasi et al. 2002]), and to commodity graphics processors. Similar diversity exists in stream-programming languages. Popular stream-programming languages include StreamC/KernelC [Rixner 2002; Mattson 2002] for the Imagine and Merrimac stream processors [Dally et al. 2003], Brook [Buck et al. 2004] for GPUs and SF95 [Yang et al. 2007] for the FT64 processor. These languages can also be used to develop stream programs for the Cell processors [Kahle et al. 2005]. Labonte et al. [2004] developed the Stream Virtual Machine and a separate compiler to improve interoperability, hence allowing development of common compilation tools and reasoning about stream program performance.

Here, we study the stream architecture that has the characteristic of SIMD stream coprocessors with a large local memory for stream buffering. The stream processors with such architecture include the Imagine stream processor, the Merrimac stream processor, GPUs, the Cell processor and the FT64 stream processor. Figure 1(b) shows a simplified diagram of such stream architecture. A stream-level program is run on the host while kernels are run on the stream processor. A single kernel that operates sequentially on records of streams in a SIMD fashion is executed on clusters of ALUs. Only data in the Local Register Files (LRFs), immediately adjacent to the arithmetic units, can be used by the clusters. Data passed to the LRFs is from the stream register file (SRF) that directly accesses off-chip memory. Off-chip memory is used for application inputs, outputs, and for intermediate streams that cannot fit in the SRF.

StreamC/KernelC [Mattson 2002] is a popular language that can be used to develop stream programs for the Imagine [Kapasi et al. 2002], Merrimac [Dally et al. 2003], Cell [Kahle et al. 2005] and FT64 [Yang et al. 2007] stream processors. Additionally, the Brook [Buck et al. 2004] language is an extension 


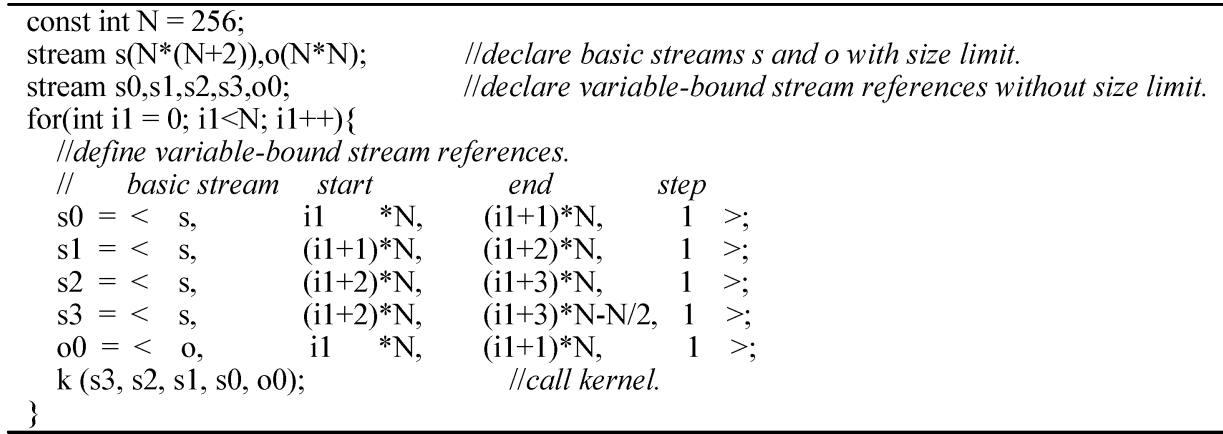

Fig. 2. Example 1, a stream program for StreamC.

of StreamC/KernelC. StreamC, an extension of $\mathrm{C}++$, is constructed by chaining stream operations together; KernelC, a C-like language, is a computationintensive function that performs computations on entire streams by applying a function to each element of the stream in sequence [Mattson 2002; Das et al. 2006]. Figure 2 shows a StreamC program. Two basic streams ( $s$ and $o$ ) and five stream references $\left(s_{0}, s_{1}, s_{2}, s_{3}\right.$, and $\left.o_{0}\right)$ are defined. Each stream reference accesses a subset of its basic stream. The stream reference is also defined by a start bound that is the index of the first record in the stream, an end bound that is the index of the record after the last record that could be in the stream, and an optional access mode that defines which records between the start and end are part of the stream. For a stream reference with constant stride access mode, its stride is used as its access mode. That is, if the access mode of a stream reference is filled in a number, it means the stream reference has constant stride access mode and the number is its stride. In Figure 2, all stream reference accesses are sequential. Stream references with constant start and end bounds are called constant-bound stream references, while the other stream references are called variable-bound stream references. Among variable-bound stream references, the most popular type of references whose start and end bounds are functions of the index variables we call loop-dependent stream references. For example, the stream reference $s_{0}$ is a loop-dependent reference and accesses the $0 t h, 1 s t, \ldots,(N-1) t h$ records of the stream $s$ on the first iteration. In order to make StreamC example code easier to understand, since StreamC is a totally new language, we assume each kernel call has a single output stream, the last parameter, in this article, although all our algorithms do not rely on this assumption and can deal with kernels with any number of outputs.

\subsection{Reusing Streams}

Memory access still dominates most stream programs' performance [Ahn et al. 2006], especially for scientific stream programs [Narayanan et al. 2002]. The only way to reduce the off-chip memory bandwidth requirement is to exploit the reuse of streams held in the SRF. Only the data defined by stream references is held sequentially in the software-managed SRF. The SRF location and length of the data are stored in a Stream Descriptor Register (SDR). Stream operations 
specify what values they operate on by referring to appropriate SDR. Except stream transfers, all operations require that the operands be taken from the $\mathrm{SRF}$ and all results be assigned to the SRF.

For two stream references accessing the same values, the second reference can reuse the values that the first reference generated in to the SRF. We call the scenario whole reuse. For two stream references accessing the shared portion of values, the second reference can reuse this part of the values generated by the first reference in to the SRF. We call the scenario partial reuse. For partial reuse, stream compilers try to find the supersequence of the two stream references, then allocate a buffer for the supersequence and allocate a SDR for each stream reference that defines which part of the buffer is accessed by the reference. For example, two input stream references $a_{0}=a(32,96)$ and $a_{1}=a(64,128)$ access the shared portion $a(64,96)$, so partial reuse exists between them. The compiler finds their supersequence, that is, $a(32,128)$, allocates a buffer for the supersequence and allocates two SDRs for the two references. One SDR describes that $a_{0}$ accesses from the $0 t h$ to $63 t h$ records of the supersequence; the other describes that $a_{1}$ accesses from the 32th to 95th records. During execution, only 96 , instead of 128 , words are loaded.

The stream compilers utilize all reuse supplied by constant-bound stream references [Mattson 2002; Mattson et al. 2004]. However, when processing a variable-bound stream reference, the stream compiler generates a stream load before each read and a stream save after each write, without utilizing any reuse.

The problem addressed in this article is to affect the reuse of data held in the SRF supplied by loop-dependent stream references. Some special consideration is required. First, the SRF is managed by software, which brings the opportunity for partial reuse. Second, moving data within the SRF is quite expensive. The SRF is divided into lanes with records interleaved among lanes so that each lane supplies data to one cluster. If stream processors, such as those with an indexed SRF and the Cell processor, allow communication across SRF lanes, interlane bandwidth is much lower than lane-to-cluster bandwidth [Jayasena 2005; Kahle et al. 2005]; if not, off-chip memory is used as a relay to avoid interlane communication by generating an SRF-to-memory and a memory-toSRF data moves. Therefore, SRF-to-SRF data moves should be avoided during the transformation. Finally, only the reuse with respect to the innermost loop is useful, due to the limited SRF capacity and the relatively large working set of kernels.

\subsection{Our Solution}

We describe an algorithm to perform a source-to-source transformation, called constant-bound stream replacement, which replaces loop-dependent stream references with constant-bound stream references to effect the reuse supplied by loop-dependent stream references. Our transformation is based on three results: lemmas identifying reuse among stream references, a new representation called the Stream Reuse Graph (SRG) and the optimization of the built SRG for the transformation. 


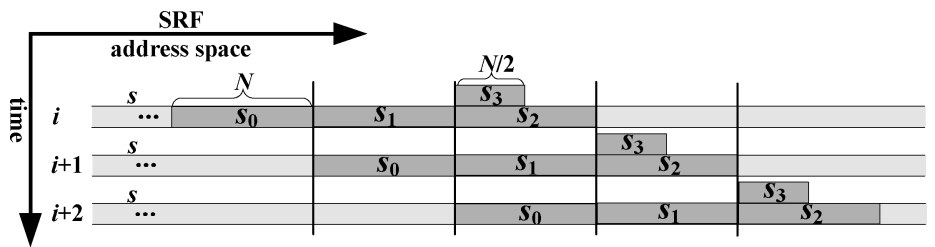

Fig. 3. Relationship of the values used by $s_{0}, s_{1}, s_{2}$, and $s_{3}$.

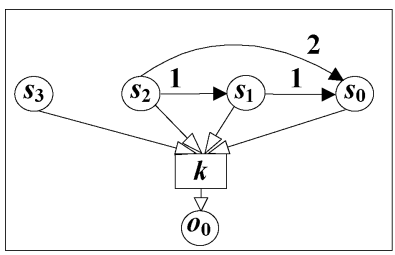

(a) SRG

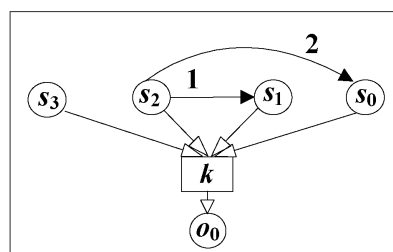

(b) Optimized SRG

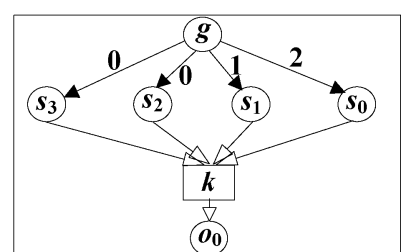

(c) Expanded SRG

Fig. 4. Building the SRG for Example 1 from Figure 2.

Our lemmas identify both the whole and partial reuse. Our algorithm first identifies the reuse of locations, then tests whether each shared location is mapped to the same lane [Mattson 2002] for stream processors that forbid interlane communication, such as Imagine, and finally judges whether the values to be reused will be unchanged, before being reused. If the values are unchanged, the reuse is possible.

The second result is a representation, SRG, which describes the whole reuse among stream references. During the SRG construction, a Directed Acyclic Graph (DAG) is built first to describe how kernels consume input streams and produce output streams. Loop-independent reuse is also depicted by the DAG. Then, a Reuse Graph (RG) is built based on the DAG, by inserting a reuse edge with the value of $d_{i, j}\left(d_{i, j} \geq 0\right)$ from the stream node $s_{i}$ to $s_{j}$ if the values generated by $s_{i}$ are used by $s_{j}$ after $d_{i, j}$ iterations.

Finally, the built SRG is optimized for the transformation from the following two aspects: (i) Because a stream node in the built SRG may have multiple possible reuse sources, we prune the SRG with the objective of making the required data loads as few as possible to select the unique factual source. The stream node, chosen as the reuse source, is called the generator. The concept is also used in [Carr and Kennedy 1994]. (ii) The SRG is expanded for partial reuse. At this step, the generator is updated to be the stream node such that other stream nodes reuse the whole or partial data that it generates before some iterations.

We use example 1 from Figure 2 to describe our solution. Figure 3 shows the relationship of the values used by $s_{0}, s_{1}, s_{2}$, and $s_{3}$. The values used by $s_{3}$ are reused as part of $s_{2}$ on the same iteration; the values used by $s_{2}$ on iteration $i$ are reused as $s_{1}$ after one iteration, reused as $s_{0}$ after two iterations. Figure 4 (to be explained in more detail later in Section 4) shows the graphs generated when building the SRG: Figure 4(a) shows the SRG that describes the whole reuse of values; Figure 4(b) shows the optimized SRG in which each stream 


\begin{tabular}{|c|c|}
\hline 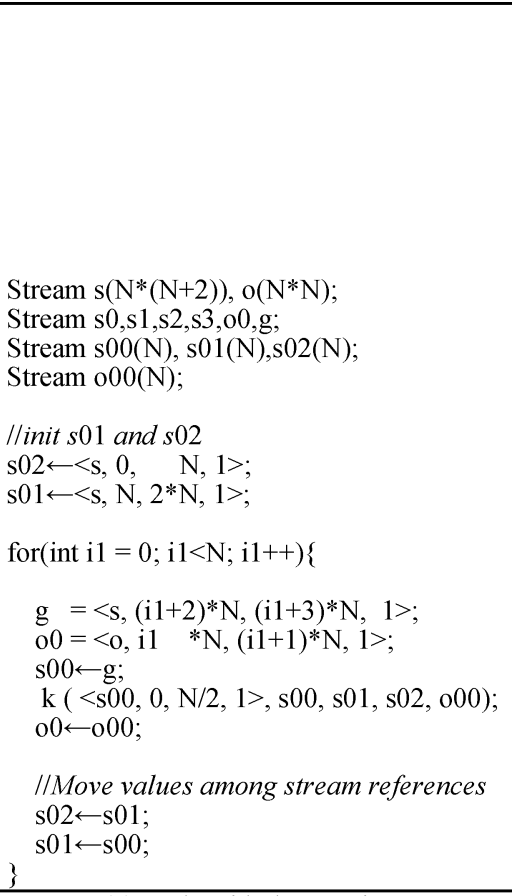 & 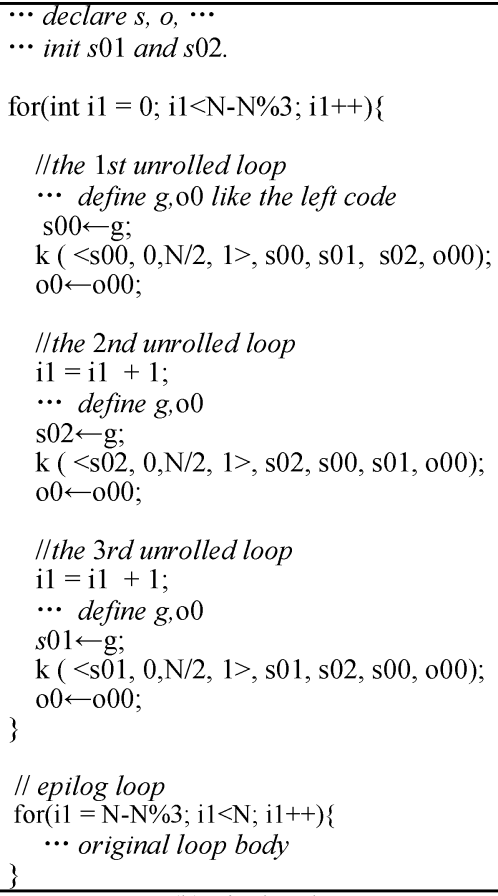 \\
\hline (a) Code with data copies & (b) Final code \\
\hline
\end{tabular}

Fig. 5. Different stages of our program transformation.

node has only one reuse source; Fig. 4(c) shows the expanded SRG with partial reuse of values. In Figure 4(c), g accesses the same sequence with $s_{2}$, and is the generator; $s_{2}, s_{1}$, and $s_{0}$ reuse the whole data generated by $g$ before zero, one, and two iterations, respectively; $s_{3}$ reuses partial data generated by $g$ on the same iteration.

We get the code in Figure 5(a) by introducing four constant-bound streams $s_{00}, s_{01}, s_{02}$, and $o_{00}$, initializing $s_{02}$ and $s_{01}$ before the loop, defining the generator $g$, loading the values generated by $g$ to $s_{00}$, replacing the references involved by references to the whole or part of the corresponding constant-bound stream references, saving the output $o_{00}$ to the locations defined by $o_{0}$, and moving the values of $s_{01}$ and $s_{00}$ to $s_{02}$ and $s_{01}$ at the end of the loop body. Since $s_{3}$ accesses from the $0 t h$ to $(N / 2) t h$ records of data generated by $g$, we replace $s_{3}$ with the reference $\left\langle s_{00}, 0, N / 2,1\right\rangle$. So, the compiler will identify and utilize both the whole and partial reuse in the example code. However, it does so at the expense of introducing two expensive SRF-to-SRF data moves. Since these moves implement a permutation of values in the SRF, we can eliminate the need for moves by unrolling to the cycle length of the permutation (equal to 3 for the example) and permuting the stream references in each unrolled loop bodies, and get the code shown in Figure 5(b).

Figure 6 graphically depicts the SRF allocation for the code before (Figure 6(a)) and after (Figure 6(b)) our transformation. Each horizontal 


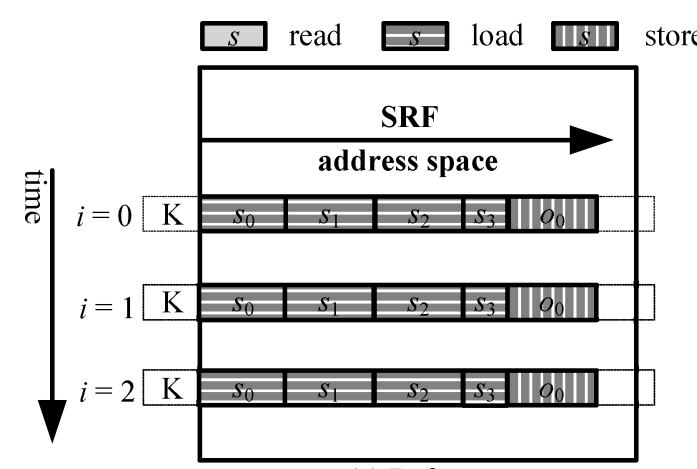

(a) Before

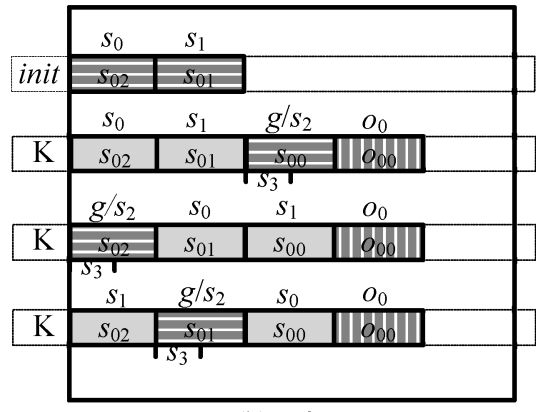

(b) After

Fig. 6. SRF allocation graph for the code before (a) and after (b) transformation.

bar indicates an iteration process, and only the first three iterations are depicted here. The large vertical bar represents the SRF. The intersection of a kernel bar and the SRF bar contains the state of the SRF for that kernel. Shaded rectangles inside such an intersection indicate a stream read or write to that portion of the SRF by that kernel. The bars with horizontal lines indicate stream loads, while those with vertical lines indicate stream stores. In the original code, the stream compiler generates a stream load for each input stream reference without utilizing any stream reuse, which brings about four stream loads on each iteration, or up to $3.5 \mathrm{~N}$ words; in the transformed code, the stream compiler generates only one stream load for the stream reference $g$, or up to $N$ words.

\subsection{Contributions}

The main contributions of this article are as follows.

-We present lemmas identifying both the whole and partial stream reuse.

- We build and optimize, for the first time, the SRG to depict stream reuse in the SRF.

-We present a program transformation, called constant-bound stream replacement, which determines the unrolling factor, unrolls the loop body and transforms the stream program to effect the reuse supplied by loop-dependent stream references.

- We build models to reduce the SRF pressure.

- We implement our method in the StreamC/KernelC compiler and evaluate the performance benefits of our techniques on a simulator for the Imagine stream processor.

Section 2 presents the work related to this article. Section 3 describes the lemmas identifying both the whole and partial stream reuse. In Section 4, we describe the construction of the SRG. Section 5 optimizes the SRG. The constant-bound stream replacement algorithm is presented in Section 6. The $\mathrm{SRF}$ pressure increased by the stream reuse exploration is considered in Section 
7. Our experiments and performance evaluation appear in Section 8, and we conclude the article and present the future in Section 9.

\section{RELATED WORK}

We divide the Related Work into groups exploiting data reuse for stream processors, earlier representations for optimizing stream programs, and exploiting data reuse supplied by indexed variables for scalar and vector processors.

Reuse exploitation for stream processors. Many schemes have been proposed to exploit the reuse of data held in the LRFs, including kernel fusion [Liao et al. 2006; Tahoori and Lee ], software-pipelining and loop unrolling, for the KernelC program [Owens et al. 2002; Das et al. 2006; Mattson et al. 2004]. These optimizations can reduce the data transferred between the SRF and LRFs, but they do not have any influence on off-chip memory usage because the LRFs transfer data only to or from the SRF. To exploit the reuse of data held in the SRF, strip-mining [Das et al. 2006], and software pipelining and loop-unrolling [Owens et al. 2002; Das et al. 2006; Mattson et al. 2004] for the StreamC program have been proposed. Although these optimizations can improve stream programs' performance, they can only be used for constantbound stream references and only loop-independent stream reuse is exploited.

To the best of our knowledge, there are no schemes that look at exploiting the reuse supplied by loop-dependent stream references. Current schemes omit all loop-carried stream reuse.

Earlier representations for optimizing stream programs. The intermediate representation stream operation precedence (SOP) graph in [Das et al. 2006] describes dependencies among stream operations and high-level control and stream data flow among basic blocks, with each supernode representing a basic block, each node in a supernode representing a stream operation, and directional edges among nodes representing the input and output streams accessed by them. With the help of the SOP graph, stream operations can be ordered to overlap as many memory transfers as possible, with all SOP dependencies satisfied. The SOP graph also can depict loop-independent reuse. However, the SOP graph cannot be directly used for the whole or partial reuse exploitation for the following reasons. First, the SOP graph was original built for constantbound stream references, so some work should be done before it is used for variable-bound stream references. Second, since the streams are denoted as directional edges; the reuse, especially for partial reuse, supplied by different stream references, is hard to depict in the SOP graph. Finally, the SOP graph cannot describe loop-carried stream reuse.

Exploiting the reuse supplied by indexed-variables for scalar and vector processors. Many researchers have demonstrated exploiting data reuse supplied by indexed-variables for scalar and vector processors. Allen and Kennedy pioneered the use of dependence to reuse the vector data in registers on vector machines [Allen 1983; Allen and Kennedy 1992]. The idea of using scalar replacement to achieve register reuse on uniprocessors was due to Carr and Kennedy [1990]. Handling complex loop nests was also due to Carr and Kennedy [1994]. 
Lu and Cooper [1997] study the impact of powerful pointer analysis in C programs for register promotion. Sastry et al. [1998] and Lo et al. [1998] show how to use static single assignment (SSA) [Cytron et al. 1991] to facilitate register promotion. Lo et al. [1998] also shows how PRE can be dualized to handle the removal of redundant store operations.

Strategies for scalar and vector reuse cannot be directly applied to exploiting the reuse supplied by loop-dependent stream references for several reasons. First, the intermediate representations, such as the dependence graph, SSA, and PRE, from which the reuse in scalar and vector processors is found and exploited, assume that data participating in the computations has the same length. However, stream references may have different lengths. Therefore, existing intermediate forms cannot be directly used to optimize stream programs. Second, partial reuse and its treatment are quite new and have never, to the best of our knowledge, appeared in scalar and vector processing. Finally, an SRF-to-SRF data move is expensive and should be avoided during program transformation.

\section{IDENTIFYING STREAM REUSE}

\subsection{Presentation}

The iteration space of an $n$-level loop nest is expressed as $\bar{i}=\left(i_{1}, \ldots, i_{n}\right)$, where $i_{1}, \ldots, i_{n}$ from left to right express the index variables from the outermost to the innermost. A stream reference of the stride type is expressed as the tuple $\langle$ name, start, end, stride $\rangle$, where name denotes its basic stream, start and end are start and end bounds, respectively, and stride is the access stride. An affine start expression is expressed as start $=\overline{v s} \cdot \bar{i}+c s$, where $\bar{i}$ is the iteration vector, $\overline{v s}$ is called the start access vector and $c s$ is the start constant term. Correspondingly, an affine end expression can be expressed as end $=\overline{v e} \cdot \bar{i}+c e$, where $\overline{v e}$ is called the end access vector and ce is the end constant term.

The reuse relationship is expressed as the tuple $\left\langle s_{0}, s_{1}, d\right\rangle$, where $d$ is called the reuse distance. If $d \geq 0, s_{1}$ reuses data generated by $s_{0}$ before $d$ iterations, with $s_{0}$ as the reuse source and $s_{1}$ as the reuse destination; otherwise, $s_{0}$ reuses the data generated by $s_{1}$ before $d$ iterations, with $s_{1}$ as the reuse source and $s_{0}$ as the reuse destination.

In addition, we assume that the innermost loop has been normalized, with $i_{n}$ increased from zero to the constant $N-1$ in steps of 1 . For simplicity, we assume here that all loops are perfectly nested.

\subsection{Stream Reuse Identification}

As with the analysis of Wolf and Lam [1991], we look to exploit reuse among stream references that have the same start access vector, end access vector and stride, and differ only in the constant terms, examples of which are shown in Example 1 in Figure 2, namely the $s_{0}, s_{1}, s_{2}$, and $s_{3}$ references. Such references are called uniformly generated stream references [Gannon et al. 1988].

Two stream references, $s_{0}=\left\langle s, \overline{v s}_{0} \cdot \bar{i}+c s_{0}, \overline{v e}_{0} \cdot \bar{i}+c e_{0}\right.$, stride $\left.e_{0}\right\rangle$ and $s_{1}=\langle s$, $\overline{v s}_{1} \cdot \bar{i}+c s_{1}, \overline{v e}_{1} \cdot \bar{i}+c e_{1}$, stride $\left._{1}\right\rangle$, are called uniformly generated stream references if (i) $\overline{v s}_{0}=\overline{v e}_{0}=\overline{v s}_{1}=\overline{v e}_{1}$, and (ii) stride $e_{0}=$ stride $_{1}$. 
As the vectors are equivalent, we use $\bar{v}$ to represent them. We also define $v_{n}$ to be the $\mathrm{n} t h$ item of $\bar{v}$. That is, $v_{n}$ represents the coefficient of the innermost index variable, $i_{n}$, in the start and end expressions.

Since little exploitable reuse exists between nonuniformly generated stream references, we partition stream references in a loop nest into equivalence classes of references that operate on the same basic stream and have the same $\bar{v}$ and stride. We call these equivalence classes uniformly generated sets [Wolf and Lam 1991]. In Example 1 from Figure 2 the stream references $s_{0}, s_{1}, s_{2}$, and $s_{3}$ are in a single uniformly generated set with a $\bar{v}$ of [1].

Next, we analyze the scenarios in which stream reuse exists. For two stream references $s_{0}$ and $s_{1}$, the reuse relationship $\left\langle s_{0}, s_{1}, d\right\rangle$ holds if the following conditions are satisfied.

(1) There are shared locations accessed by $s_{0}$ on iteration $i_{n}$ and by $s_{1}$ on iteration $i_{n}+d$.

(2) Each shared location must be mapped to the same SRF lane [Mattson 2002] for stream processors that forbid interlane communication, such as Imagine. This condition is not necessary for stream processors with an indexed SRF [Jayasena et al. 2004] and the Cell processor because these processors allow interlane communication.

(3) The values generated by the reuse source should not be changed in the next $d$ iterations.

Condition (1) is satisfied in the following scenarios. An innermost loopinvariant reference (i.e., $v_{n}=0$ ) accesses the same locations in iterations with the same outer loop indice but different innermost loop indice; a stream reference reuses the values in the SRF generated by another reference on previous iterations (or by previous reference on the same iteration). Lemma 3.1 judges whether Condition (1) is satisfied for $s_{0}$ and $s_{1}$.

LEMMA 3.1. For two stream references $s_{0}$ and $s_{1}$ in the same uniformly generated set, there are shared locations accessed by $s_{0}$ on iteration $i_{n}$ and by $s_{1}$ on iteration $i_{n}+d$ if:

(a) if $v_{n}=0,\left(\left(\min \left\{c e_{0}, c e_{1}\right\}-\max \left\{c s_{0}, c s_{1}\right\}\right) \geq 1\right) \&\left(\left(c s_{0}-c s_{1}\right) \bmod\right.$ stride $\left.=0\right)$;

(b) otherwise, $\left(\left(\min \left\{c e_{0}-c s_{0} / v_{n} \times v_{n}, c e_{1}-c s_{1} / v_{n} \times v_{n}\right\}-\max \left\{c s_{0} \bmod v_{n}\right.\right.\right.$, $\left.\left.\left.c s_{1} \bmod v_{n}\right\}\right) \geq 1\right) \& \&\left(\left(c s_{0} \bmod v_{n}-c s_{1} \bmod v_{n}\right) \bmod\right.$ stride $\left.=0\right)$.

PRoof. Since an innermost loop-invariant stream reference accesses the same memory locations at the iterations with the same outer loop indices but different innermost indices, the innermost loop-invariant stream references $s_{0}$ and $s_{1}$ access their possible shared locations at every such iteration. Let base $e_{s}$ be the start address of $s$, so memory locations accessed by $s_{0}$ on iteration $i_{n}$ can be expressed as Mem $_{0, i_{n}}=\left[\right.$ base $_{s}+\bar{v} \cdot \overline{i_{n}}+c s_{0}$, base $_{s}+\bar{v} \cdot \overline{i_{n}}+c e_{0}$, stride), those by $s_{1}$ on iteration $i_{n}$ as Mem $_{1, i_{n}}=\left[\right.$ base $_{s}+\bar{v} \cdot \overline{i_{n}}+c s_{1}$, base $_{s}+\bar{v} \cdot \overline{i_{n}}+c e_{1}$, stride), and their possible shared locations as Mem shared $=\left[\right.$ base $_{s}+\bar{v} \cdot \overline{i_{n}}+\max \left\{c s_{0}, c s_{1}\right\}$, base $_{s}+\bar{v} \cdot \overline{i_{n}}+\min \left\{c e_{0}, c e_{1}\right\}$, stride). For $s_{0}$ and $s_{1}$ such that satisfy the formula in $(\mathrm{a}),\left(\right.$ Mem $_{\text {shared }}$.end-Mem shared.$\left.s t a r t\right)=\left(\min \left\{c e_{0}, c e_{1}\right\}-\max \left\{c s_{0}, c s_{1}\right\}\right) \geq 1$. This means Mem $_{\text {shared }}$ has more than one record. Assuming that $c s_{0} \bmod$ stride $=m$, 


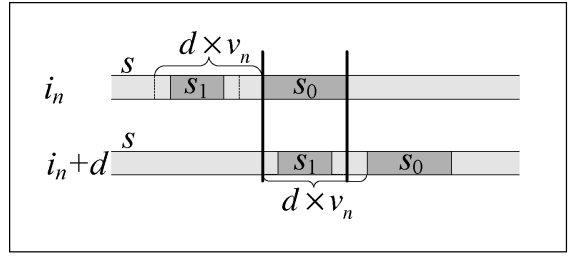

(a) $s_{0}$ reused as $s_{1}$ after $d$ iterations

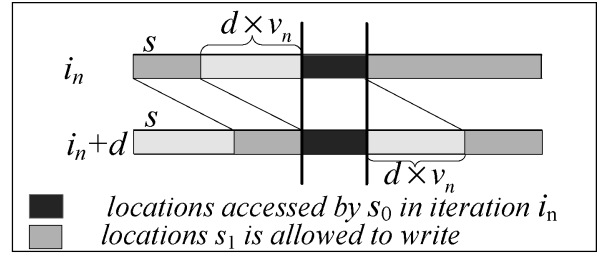

(b) Scenario that $s_{0}$ is unchanged by $s_{1}$ in the next $d$ iterations

Fig. 7. Example for Lemmas 1 and 3.

the start of Mem $_{\text {shared }}$ is accessed by both $s_{0}$ and $s_{1}$ because $\left(c s_{0}-c s_{1}\right) \bmod$ stride $=0$, thus $c s_{1} \bmod$ stride $=m$, and thus $\max \left\{c s_{0}, c s_{1}\right\} \bmod$ stride $=m$. This means $M_{\text {em }}$ shared is accessed by both $s_{0}$ and $s_{1}$. Therefore, innermost loop-invariant stream references such that satisfy the formula in (a) have shared locations. And we let $d=0$ denote such scenario.

If $v_{n} \neq 0$, let $d=\left(c s_{0} / v_{n}-c s_{1} / v_{n}\right)$. Thus, the memory locations accessed by $s_{0}$ on iteration $i_{n}$ can be expressed as Mem $_{0, i_{n}}=\left[\right.$ base $_{s}+\bar{v} \cdot \overline{i_{n}}+c s_{0}$, base $_{s}+\bar{v} \cdot \overline{i_{n}}+c e_{0}$, stride), those by $s_{1}$ on iteration $i_{n}+d$ as Mem $_{1, i_{n}+d}=\left[\right.$ base $_{s}+\bar{v} \cdot \overline{i_{n}}+c s_{1}+c s_{0} / v_{n} \times$ $v_{n}-c s_{1} / v_{n} \times v_{n}$, base $_{s}+\bar{v} \cdot \overline{i_{n}}+c e_{1}+c s_{0} / v_{n} \times v_{n}-c s_{1} / v_{n} \times v_{n}$, stride), and their possible shared locations as Mem shared $=\left[\right.$ base $_{s}+\bar{v} \cdot \overline{i_{n}}+\max \left\{c s_{0}, c s_{1}+c s_{0} / v_{n} \times\right.$ $\left.v_{n}-c s_{1} / v_{n} \times v_{n}\right\}$, base $_{s}+\bar{v} \cdot \overline{i_{n}}+\min \left\{c e_{0}, c e_{1}+c s_{0} / v_{n} \times v_{n}-c s_{1} / v_{n} \times v_{n}\right\}$, stride $)$. For $s_{0}$ and $s_{1}$ such that satisfy the formula in (b), $\left(\right.$ Mem $_{\text {shared }}$. end - Mem $_{\text {shared }}$. start $)=$ $\min \left\{c e_{0}, c e_{1}+c s_{0} / v_{n} \times v_{n}-c s_{1} / v_{n} \times v_{n}\right\}-\max \left\{c s_{0}, c s_{1}+c s_{0} / v_{n} \times v_{n}-c s_{1} / v_{n} \times v_{n}\right\}$. If $c s_{0} \geq\left(c s_{1}+c s_{0} / v_{n} \times v_{n}-c s_{1} / v_{n} \times v_{n}\right),\left(M_{\text {sem }}\right.$ shared $\left.. e n d-M e m_{\text {shared }} . s t a r t\right) \geq 1$ because $\left(c e_{0}-c s_{0}\right) \geq 1$ and $\left(c e_{1}+c s_{0} / v_{n} \times v_{n}-c s_{1} / v_{n} \times v_{n}-c s_{0}\right)=\left(c e_{1}-\right.$ $\left.c s_{1} / v_{n} \times v_{n}-c s_{0} \bmod v_{n}\right) \geq 1$. Similarly, $\left(\right.$ Mem $_{\text {shared }} . e n d-$ Mem $_{\text {shared }}$. start $) \geq 1$ if $c s_{0}<\left(c s_{1}+c s_{0} / v_{n} \times v_{n}-c s_{1} / v_{n} \times v_{n}\right)$.This means $M_{\text {sem }}$ shared has more than one record. Assuming that $c s_{0} \bmod$ stride $=m$, the start of Mem shared $_{\text {is accessed }}$ by both $s_{0}$ and $s_{1}$ because $\left(c s_{0} \bmod v_{n}-c s_{1} \bmod v_{n}\right) \bmod$ stride $=0$, thus $\left(c s_{1}+c s_{0} / v_{n} \times v_{n}-c s_{1} / v_{n} \times v_{n}\right) \bmod$ stride $=\left(c s_{0}+\left(c s_{1} \bmod v_{n}-c s_{0} \bmod v_{n}\right)\right) \bmod$ stride $=c s_{0} \bmod$ stride $=m$, and thus $\max \left\{c s_{0}, c s_{1}+c s_{0} / v_{n} \times v_{n}-c s_{1} / v_{n} \times v_{n}\right\}$ $\bmod$ stride $=m$. This means $M e m_{\text {shared }}$ is accessed by both $s_{0}$ and $s_{1}$. Therefore, for innermost loop-variant stream references $s_{0}$ and $s_{1}$ such that satisfy the formula in (b), there are shared locations accessed by $s_{0}$ on iteration $i_{n}$ and by $s_{1}$ on iteration $i_{n}+d$ with $d=\left(c s_{0} / v_{n}-c s_{1} / v_{n}\right)$.

To unify the process of innermost loop-invariant and loop-variant stream references, we define $C=\max \left\{1, v_{n}\right\}$, equal to 1 if $v_{n}=0$, otherwise equal to $v_{n}$. Thus, formulas in (a) and (b) can be merged into one as follows:

$\left(\left(\min \left\{c e_{0}-c s_{0} / C \times v_{n}, c e_{1}-c s_{1} / C \times v_{n}\right\}-\max \left\{c s_{0} \bmod C, c s_{1} \bmod C\right\}\right) \geq 1\right) \&$ $\left(\left(c s_{0} \bmod C-c s_{1} \bmod C\right) \bmod\right.$ stride $\left.=0\right)$.

If $c s_{0}=c s_{1}+c s_{0} / C \times v_{n}-c s_{1} / C \times v_{n}$ and $c e_{0}=c e_{1}+c s_{0} / C \times v_{n}-c s_{1} / C \times v_{n}$, the whole location reuse exists between $s_{0}$ and $s_{1}$; otherwise, partial location reuse exists. If $d \geq 0$, then $s_{0}$ is the reuse source and $s_{1}$ is the reuse destination; otherwise, $s_{1}$ is the reuse source and $s_{0}$ is the reuse destination.

Figure 7(a) shows the stream references $s_{0}$ and $s_{1}$ that meet the conditions of Lemma 3.1 with $d>0, v_{n}>0$, and stride $=1$. As shown, part of the locations 
accessed by $s_{0}$ on iteration $i_{n}$ are accessed by $s_{1}$ on iterations $i_{n}+d$, hence the reuse.

For Condition (2), how the shared locations are mapped to the SRF totally depends on the SRF address mode. In the Imagine stream processor that does not support interlane communication, data used by each stream reference on every iteration is mapped from lane 0 , with data interleaved at one-record granularity among the SRF lanes [Jayasena 2005]. Lemma 2 takes the address mapping mode of the Imagine stream processor and judges whether each of the shared locations identified by Lemma 1 is mapped to the same SRF lane by $s_{0}$ and $s_{1}$.

Lemma 2. For two stream references $s_{0}$ and $s_{1}$ in the same uniformly generated set, each of the shared locations accessed by $s_{0}$ on iteration $i_{n}$ and by $s_{1}$ on iteration $i_{n}+d$ is mapped to the same $S R F$ lane if $\left(c s_{0}-c s_{1}\right) /$ stride $\bmod N C=0$, where NC denotes the number of clusters.

PRoof. In the Imagine stream processor, the record $\bar{v} \cdot \overline{i_{n}}+r_{0}$ accessed by $s_{0}$ is mapped to the $\left(\left(\left(r_{0}-c s_{0}\right) /\right.\right.$ stride $\left.) \bmod N C\right)$-th lane, and " $\bar{v} \cdot \overline{i_{n}}+r_{1}$ " by $s_{1}$ to the $\left(\left(\left(r_{1}-c s_{1}\right) /\right.\right.$ stride $\left.) \bmod N C\right)-t h$ lane. As discussed earlier, the shared locations accessed by $s_{0}$ on iteration $i_{n}$ and $s_{1}$ on iteration $i_{n}+d$ can be expressed as Mem $_{\text {shared }}=\left[\right.$ base $_{s}+\bar{v} \cdot \overline{i_{n}}+\max \left\{c s_{0}, c s_{1}+c s_{0} / C \times v_{n}-c s_{1} / C \times v_{n}\right\}$, base $_{s}+\bar{v}$. $\overline{i_{n}}+\min \left\{c e_{0}, c e_{1}+c s_{0} / C \times v_{n}-c s_{1} / C \times v_{n}\right\}$, stride). If $c s_{0} \geq c s_{1}+c s_{0} / C \times v_{n}-$ $c s_{1} / C \times v_{n}$, the first shared address is mapped to the $0 t h$ lane by $s_{0}$ and to the $\left(\left(\left(c s_{0}-c s_{1}\right) /\right.\right.$ stride $\left.) \bmod N C\right)$-th lane by $s_{1}$. If and only if $\left(\left(c s_{0}-c s_{1}\right) /\right.$ stride $) \bmod$ $N C=0$, it is also mapped to lane 0 by $s_{1}$. Similarly, the conclusion holds if $c s_{0}<c s_{1}+c s_{0} / C \times v_{n}-c s_{1} / C \times v_{n}$.

Since the reuse with respect to the SRF is the reuse of values, intervening writes introduce output dependencies, potentially inhibiting reuse. If the locations to be reused are not written to before reuse occurs, then reusing is possible. Lemma 3 judges whether the values generated by the reuse source $s_{0}$ are not changed by the output reference $s_{1}$ in the next $d$ iterations.

Lemma 3. For two stream references $s_{0}$ and $s_{1}$ in the same uniformly generated set, the values used by $s_{0}$ are not changed by the output stream reference $s_{1}$ in the next $d(d>0)$ iterations, if $c e_{0}<c s_{1}+\min \left\{0, v_{n} \times d\right\} \| c s_{0}>c e_{1}+\max \left\{0, v_{n} \times d\right\}$.

Proof. As proof of Lemma 1, the possible shared locations accessed by $s_{0}$ at iteration $i_{n}$ and $s_{1}$ at iterations $i_{n}, \ldots, i_{n}+d$ can be expressed as $M e m_{\text {shared }}=$ $\left[\right.$ base $_{s}+\bar{v} \cdot \overline{i_{n}}+\max \left\{c s_{0}, c s_{1}+\{0, \ldots, d\} \times v_{n}\right\}$, base $_{s}+\bar{v} \cdot \overline{i_{n}}+\min \left\{c e_{0}, c e_{1}+\{0, \ldots, d\} \times\right.$ $\left.v_{n}\right\}$, stride). If $c s_{0} \geq\left(c s_{1}+\{0, \ldots, d\} \times v_{n}\right), M_{\text {sem }}$ shared has no records if and only if "cs $0>c e_{1}+\{0, \ldots, d\} \times v_{n}$ ", that is, "cs $s_{0}>c e_{1}+\max \left\{0, v_{n} \times d\right\}$ "; otherwise, $M e m_{\text {shared }}$ has no records if and only if "ce $e_{0}<c s_{1}+\{0, \ldots, d\} \times v_{n}$ ", that is, “ce $e_{0}<c s_{1}+\min \left\{0, v_{n} \times d\right\}$ ".

Figure 7(b) draws the locations accessed by $s_{0}$ on iteration $i_{n}$ and the locations that $s_{1}$ is allowed to write by Lemma 3 with $v_{n}>0$. As shown, the values used by $s_{0}$ on iteration $i_{n}$ are not changed by $s_{1}$ in the next $d$ iterations. 


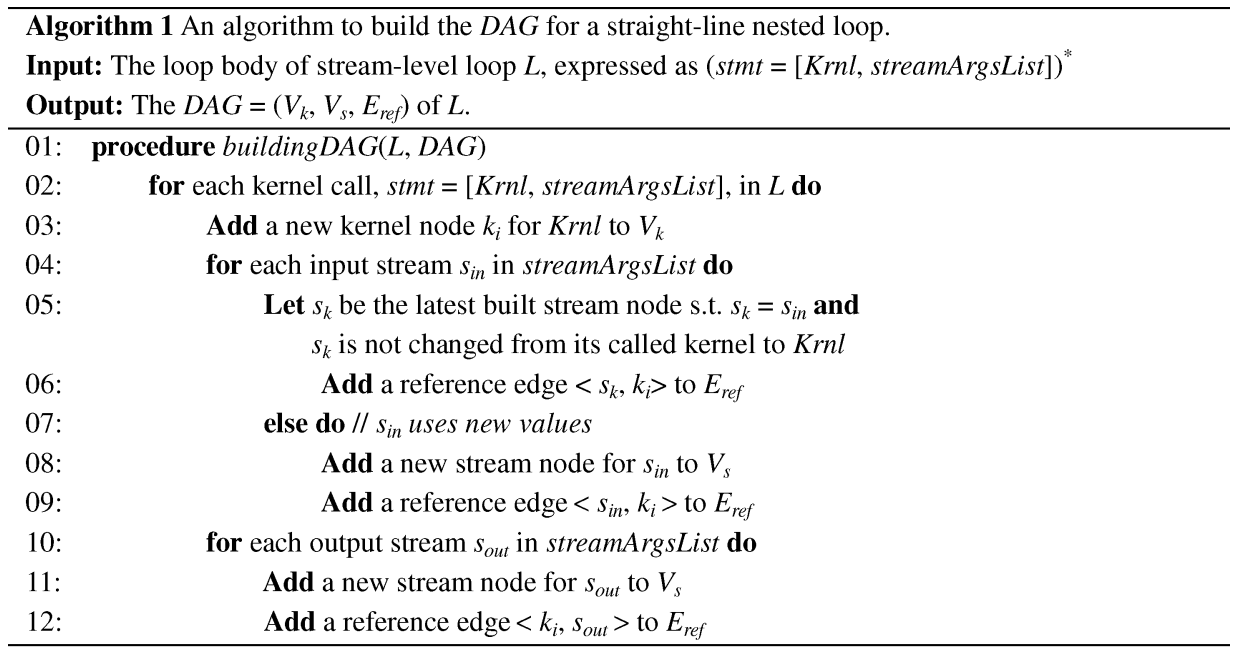

Fig. 8. Algorithm building the DAG for a straight-line nested loop.

\section{STREAM REUSE GRAPH}

The SRG captures value reuse supplied by stream references and is applicable across differing stream architectures. It consists of the following components: stream nodes, kernel nodes, reference edges, and reuse edges. The stream node, drawn as a circle, denotes a stream reference with distinct values. The kernel node, drawn as a square block, denotes a kernel call. The directional reference edge, expressed as a hollow arrow, depicts that a kernel consumes an input stream (pointing from the stream node to the kernel node) or produces an output stream (the arrow comes from the kernel node). The directional reuse edge, drawn as a solid arrow with its value $d$, shows values of the source node are reused as the destination after $d$ iterations. Stream nodes, kernel nodes, and reference edges form a $D A G$ that captures the reference relationship among kernels and stream nodes. Stream nodes and reuse edges form a $R G$ that captures the reuse relationship among stream nodes. The standard SRG depicts only the whole stream reuse. Section 5.2 will extend the SRG to also depict partial reuse.

The standard SRG is built for the straight-line loop $L$ in the following two steps. An extension of this work is to consider branches within the innermost loop. The SRG is still applicable as branches can be restructured to be around the innermost loops.

Building the DAG. This step builds the DAG, describing the relationship between stream references and kernels, for $L$; it builds kernel nodes and stream nodes, and then inserts reference edges between them. During the construction, a stream node is built only for the stream reference with distinct values. That is, if two stream references use the same values, only one stream node is built for the first reference, and a reference edge is built from the stream node to the kernel that involves the second reference, so the DAG also describes loopindependent reuse. 


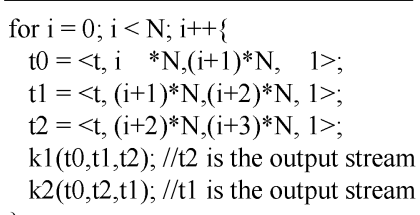

(a) Example 2 .

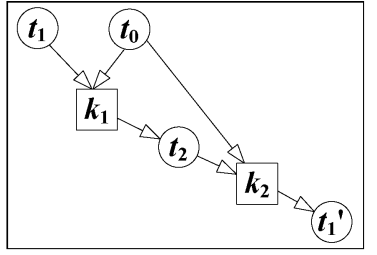

(b) DAG.

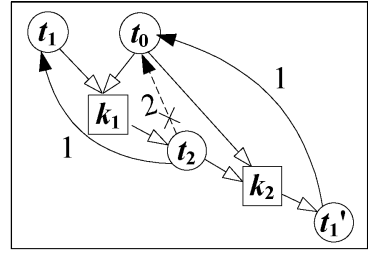

(c) SRG.

Fig. 9. Example 2 showing the construction of the SRG.

Algorithm 1 in Figure 8 builds the DAG for a stream-level loop. First, a kernel node $k_{i}$ is built for the corresponding ith kernel call Krnl (line 3). Next stream references within $k_{i}$ are processed (lines 4-12). Algorithm 1 builds a stream node for the stream reference with distinct values (lines 7-12). Such a stream reference is either loaded from off-chip memory if accessed for the first time, or produced by Krnl. For the other references, Algorithm 1 finds the stream node generating data needed by the reference (lines 5-6).

In Algorithm 1, an output stream node is created for each output reference (lines 10-12). However, while dealing with an input reference $s_{i n}$, this step searches all built stream nodes for stream nodes accessing the same locations as $s_{i n}$, by examining whether they have the same access vector, start constant term, end constant term, and stride. The latest built node, $s_{k}$, of the found nodes has the values needed by the input reference if its data is not changed from its building to calling $K r n l$, by testing whether there is a stream node, built during this period, accessing any locations defined by $s_{k}$ at the same iteration (lines $5-6)$. If $s_{k}$ is not found or its data is changed during this period, an input stream node is built for $s_{i n}$ (lines 7-9). Finally, reference edges are inserted from the built or found stream nodes to the kernel node $k_{i}$.

The DAG for the code in Figure 9(a), for example, is built as follows. The kernel node $k_{1}$ is built first; the stream nodes $t_{0}, t_{1}$, and $t_{2}$ are built because they generate new distinct values. Next, the kernel node $k_{2}$ is built. However, because the values needed by its input reference $t_{0}$ have been loaded into the SRF by the built stream node $t_{0}$, a reference edge is inserted from $t_{0}$ to $k_{2}$. Similar treatment occurs for its input reference $t_{2}$. A stream node, $t_{1}^{\prime}$, is built for $k_{2}$ 's output stream reference $t_{1}$, since $k_{2}$ produces new values for it. The built DAG is shown in Figure 9(b).

The DAG describes the definition and use of the stream references in $L$. As a result, loop-independent reuse is shown in the DAG in the form of two or more kernel nodes being connected to the same stream node.

Building reuse edges. This step builds the reuse edges among stream nodes based on the built DAG. It identifies all incidents of loop-carried whole value reuse among stream nodes and adds corresponding reuse edges. Algorithm 2 in Figure 10 finishes this job.

As each innermost loop-invariant stream reference accesses the same locations on the innermost iterations with the same outer loop indice but different innermost loop indice, location reuse exists between each reference to itself. For such a read reference, $s_{i}$, if there is not any output stream reference 


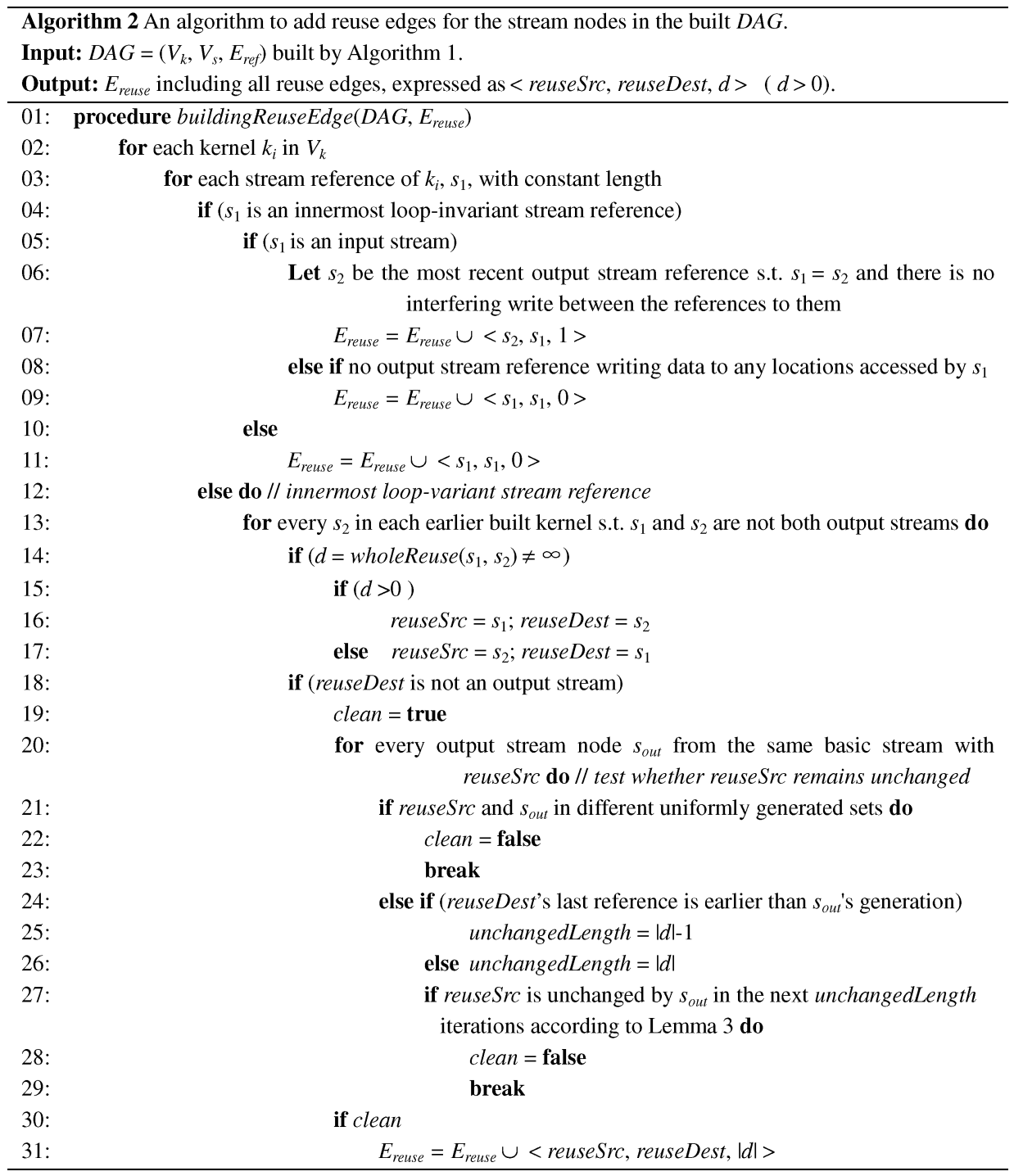

Fig. 10. Algorithm adding reuse edges for the stream nodes in the built DAG.

writing data to any locations accessed by $s_{i}$ after the needed data is generated by $s_{i}$ on the first innermost iteration, $s_{i}$ can reuse the data on the rest of such iterations. Therefore, the number of data loads for $s_{i}$ can be reduced from $N$ to 1 by loading the data into a constant-bound stream reference, just before the innermost loop, and replacing the reference to $s_{i}$ with that to the constant-bound stream reference. However, if a write reference generates the wanted data for the same locations and the data remains unchanged until the reference to $s_{i}$, $s_{i}$ can reuse the data. Similarly, for an innermost loop-invariant write stream reference, $s_{o}$, only the data generated by $s_{o}$ on the last innermost iteration is needed to write back to off-chip DRAM because $s_{o}$ writes data to the same 
locations on every such innermost iteration. Therefore, the number of data loads for $s_{o}$ can be reduced from $N$ to 1 by saving the data to a constant-bound stream reference, just after the innermost loop, and replacing the reference to $s_{o}$ with that to the constant-bound stream reference.

Therefore, for the innermost loop-invariant input reference, $s_{1}$, Algorithm 2 first examines whether there is a write reference, $s_{2}$, generating data for the addresses accessed by $s_{1}$ and the data remains unchanged until the first reference to $s_{1}$ : if present, Algorithm 2 adds a reuse edge from $s_{2}$ to $s_{1}$ with the value equal to 1 (lines 6-7); otherwise, Algorithm 2 adds a reuse edge from $s_{1}$ to itself with the value equal to 0 if the locations accessed by $s_{1}$ are not changed by any output stream reference (lines 8-9). However, if $s_{1}$ is an innermost loop-invariant output reference, Algorithm 2 adds a reuse edge from $s_{1}$ to itself (lines 10-11).

If $s_{1}$ is an innermost loop-variant stream reference (lines 12-31), Algorithm 2, based on Lemma 1 (with Lemma 2 for stream processors forbidding interlane communication), identifies the opportunities for the whole location reuse supplied by $s_{1}$ and another earlier built stream node $s_{2}$, computes their reuse distance, $d$, and finds the reuse source and destination (lines 13-17). Then, it detects whether the values of the reuse source are not changed by any output stream node $s_{\text {out }}$ from the same basic stream before the reuse (lines 18-29). The values of the reuse source are regarded as changed by $s_{\text {out }}$ if they are in different uniformly generated sets (lines 21-23). Otherwise, if the reuse destination is a parameter of an earlier built kernel than the first kernel call referring to $s_{\text {out }}$, this step, based on Lemma 3, tests whether the values remain unchanged in next $|d|-1$ iterations (line 25). (If $|d|-1 \leq 0$, the test is not necessary.); if not, the algorithm tests whether the values remain unchanged in the next $|d|$ iterations (line 26). If the reuse source remains unchanged before the reuse, the value reuse $\left\langle s_{0}, s_{1}, d\right\rangle$ holds. A reuse edge is inserted from the reuse source to the destination with $|d|$ as its value (lines 30-31).

The graph in Figure 9(c) is built by this step. The values of $t_{2}$ are changed by the output stream node $t_{1}^{\prime}$ after one iteration. Therefore, $t_{2}$ cannot be reused as $t_{0}$ after two iterations. However, a reuse edge is inserted from $t_{2}$ to $t_{1}$ because the change to the values occurs after the reuse.

\section{OPTIMIZING THE SRG}

Although the built SRG describes all whole reuse in loop $L$, it cannot be used for the transformation for the following reasons.

(1) One stream node may have multiple possible data sources, such as the stream node $s_{0}$ in the SRG in Figure 4(a) that is built for Example 1 from Figure 2. In fact, each stream node has only one factual reuse source that generates the data used by the stream node. Since only the factual reuse source needs memory transfers, we should choose it for each stream node in order to reduce as many memory accesses as possible.

(2) The SRG does not describe the partial reuse.

Next, we improve the built SRG to overcome these deficiencies. 


\subsection{Selecting Reuse Source}

Based on the SRG properties, we choose the factual reuse source with the objective of making the required data loads as few as possible. The SRG properties, stated in Lemmas 4 and 5, are first discussed before the reuse source is selected.

LEMMA 4. If $\left\langle s_{1}, s_{2}, d_{1,2}\right\rangle\left(d_{1,2} \geq 0\right)$ and $\left\langle s_{2}, s_{3}, d_{2,3}\right\rangle\left(d_{2,3} \geq 0\right)$ are satisfied, $\left\langle s_{1}, s_{3}, d_{1,2}+d_{2,3}\right\rangle$ holds.

Proof. Let the memory locations accessed by $s_{3}$ on iteration $i_{n}$ be [Addr $r_{s}, A d d r_{e}$, stride). Then, the memory locations accessed by $s_{3}$ on iteration $i_{n}+d_{2,3}$ are expressed as [Addr $r_{s}+v_{n} \times d_{2,3}, A d d r_{e}+v_{n} \times d_{2,3}$, stride). Because $\left\langle s_{2}, s_{3}, d_{2,3}\right\rangle$ holds, the locations accessed by $s_{2}$ on iteration $i_{n}$ are the same with those accessed by $s_{3}$ on iteration $i_{n}+d_{2,3}$, that is, [Addr $r_{s}+v_{n} \times d_{2,3}, A d d r_{e}+$ $v_{n} \times d_{2,3}$, stride). Therefore, the locations accessed by $s_{2}$ on iteration $i_{n}+d_{1,2}$ are expressed as $\left[A d d r_{s}+v_{n} \times d_{2,3}+v_{n} \times d_{1,2}, A d d r_{e}+v_{n} \times d_{2,3}+v_{n} \times d_{1,2}\right.$, stride), which are the locations accessed by $s_{3}$ on iteration $i_{n}+d_{1,2}+d_{2,3}$, and are also the locations accessed by $s_{1}$ on iteration $i_{n}$ because $\left\langle s_{1}, s_{2}, d_{1,2}\right\rangle$ holds. So, the location reuse $\left\langle s_{1}, s_{3}, d_{1,2}+d_{2,3}\right\rangle$ holds. Because the data generated by $s_{1}$ is reused as $s_{2}$ after $d_{1,2}$ iterations and that generated by $s_{2}$ is reused as $s_{3}$ after $d_{2,3}$ iterations, the data used by $s_{2}$ on iteration $i_{n}$, which is generated by $s_{1}$ before $d_{1,2}$ iterations, remains unchanged in the next $d_{2,3}$ iterations. That is, the data generated by $s_{1}$ remains unchanged in the next $d_{1,2}+d_{2,3}$ iterations. Therefore, the value reuse $\left\langle s_{1}, s_{3}, d_{1,2}+d_{2,3}\right\rangle$ holds.

Lemma 5. If $\left\langle s_{1}, s_{2}, d_{1,2}\right\rangle\left(d_{1,2} \geq 0\right)$ and $\left\langle s_{1}, s_{3}, d_{1,3}\right\rangle\left(d_{1,3} \geq 0\right)$ are satisfied, $\left\langle s_{2}, s_{3}, d_{1,2}-d_{1,3}\right\rangle$ holds.

Proof. The proof is similar to Lemma 4. We omit it here.

Based on basic graph theory [West 2001], the RG can be partitioned into $q$ subgraphs, called sub-RGs, where there is no reuse edge connecting the stream nodes in different sub-RGs. That is, for stream nodes $s_{1}\left(s_{1} \in s u b-R G_{1}\right)$ and $s_{2}$ $\left(s_{2} \in s u b-R G_{2}\right)$, there is no reuse edge between them, that is, $\left\langle s_{1}, s_{2}\right\rangle \notin E_{\text {ref }}$ and $\left\langle s_{2}, s_{1}\right\rangle \notin E_{\text {ref }}$.

THEOREM 1. There is one and only one stream node that acts as the reuse source of every other stream node in each sub-RG $G_{i}$. That is, there is a reuse edge issued from the stream node to every other stream node and no reuse edge issued from any other stream node to it.

Proof. We prove existence first. Obviously, for $s u b-R G_{i}$ only having one or two stream nodes, the conclusion is reached. When there are three stream nodes, $s_{1}, s_{2}$, and $s_{3}$, assuming, without the loss of generality, that $\left\langle s_{2}, s_{1}, d_{2,1}\right\rangle \in$ $E_{r e f}$, one of the four following relations is satisfied: (i) $\left\langle s_{2}, s_{3}, d_{2,3}\right\rangle \in E_{\text {ref }}$; (ii) $\left\langle s_{1}, s_{3}, d_{1,3}\right\rangle \in E_{r e f}$; (iii) $\left\langle s_{3}, s_{2}, d_{3,2}\right\rangle \in E_{r e f}$; or (4) $\left\langle s_{3}, s_{1}, d_{3,1}\right\rangle \in E_{r e f}$. When (i) is satisfied, $s_{2}$ is the wanted stream node. When (ii) is satisfied, $\left\langle s_{2}, s_{3}, d_{2,1}+d_{1,3}\right\rangle \in$ $E_{\text {ref }}$ holds based on Lemma 4 . So, $s_{2}$ is the wanted stream node. When (iii) is satisfied, $\left\langle s_{3}, s_{1}, d_{3,2}+d_{2,1}\right\rangle \in E_{\text {ref }}$ holds based on Lemma 4 . So, $s_{3}$ is the wanted stream node. When (iv) is satisfied, $\left\langle s_{2}, s_{3}, d_{2,1}-d_{3,1}\right\rangle$ holds based on Lemma 5 . If $d_{2,1}>d_{3,1}, s_{2}$ is the wanted node; otherwise, $s_{3}$ is the wanted node. We assume 
that the conclusion that one stream node in $s u b-R G_{i}$ is the reuse source of all the other stream nodes is reached when there are $N$ stream nodes in $s u b-R G_{i}$. When there are $N+1$ stream nodes in $s u b-R G_{i}$, except a certain stream node $s_{1}$, the other $N$ stream nodes with the reuse edges among them compose a new graph, denoted $s u b-R G$ '. Because $s u b-R G$ ' has $N$ stream nodes, we can conclude that there is a stream node acting as the others' reuse source, we call it $s_{2}$. There must be a reuse edge between $s_{1}$ and some stream node $s_{3}$ in sub-RG'. That is, $\left\langle s_{3}, s_{1}, d_{3,1}\right\rangle \in E_{\text {ref }}$ or $\left\langle s_{1}, s_{3}, d_{1,3}\right\rangle \in E_{\text {ref }}$ holds. If the first holds, $\left\langle s_{2}, s_{1}, d_{2,3}+d_{3,1}\right\rangle \in E_{r e f}$ holds, based on Lemma 4 . So $s_{2}$ is the wanted stream node. Otherwise, $\left\langle s_{2}, s_{1}, d_{2,3}-d_{1,3}\right\rangle$ holds based on Lemma 5 . If $d_{2,3}>d_{1,3}, s_{2}$ is the wanted stream node; otherwise, $s_{1}$ is the wanted stream node.

Next, we prove uniqueness. Supposing that there are two different stream nodes, $s$ and $s^{\prime}$, meeting the condition of the theorem, $\left\langle s, s^{\prime}, d\right\rangle \in E_{r e f}$ and $\left\langle s^{\prime}, s, d^{\prime}\right\rangle \in E_{r e f}$ hold. That is, $s^{\prime}$ can reuse data generated by $s$ before $d$ iterations and $s$ can reuse data generated by $s^{\prime}$ before $d^{\prime}$ iterations, which can be satisfied only when (i) $s$ and $s^{\prime}$ are stream references accessing the same locations, and (ii) the data generated by one stream node remains unchanged before the other one reuses it. However, Algorithm 1 only builds one stream node for two such stream references. Therefore, $s$ and $s^{\prime}$ are the same stream node.

We call the stream node defined by Theorem 1 the generator of a sub-RG. This is because all other stream nodes in the $s u b-R G$ can reuse the values generated by this stream node before some iterations. This concept comes from Callahan et al. [1990]. We choose the generator, denoted $g_{i}$, as the data source for the stream node $s_{i, j}$ in $s u b-R G_{i}$. So only $g_{i}$ requires memory transfers, while $s_{i, j}$ reuses the values generated by $g_{i}$ before $d_{i, j}$ iterations. Otherwise, at least two stream transfers are necessary for $s u b-R G_{i}$ with any other stream node selected as the reuse source. Let $D_{i}$ be the maximum reuse distance in $s u b-R G_{i}$, and for $s u b-R G_{i}$, the total number of stream transfers is reduced from $D_{i}$ to 1 on each iteration.

Therefore, in this step, we find the generator for $s u b-R G_{i}$ and cut any reuse edge issued from other stream node than the generator. Algorithm 3 in Figure 11 finishes this job. First, it picks up sub-RG $($ line 20), then finds the generator $g_{i}$ for $s u b-R G_{i}$ (line 21) and finally cuts all reuse edges that are not issued from $g_{i}$ in $s u b-R G_{i}$ (line 22). This processing is repeated until all $s u b$ $R G s$ are found (lines 18-25). When picking up a sub-RG (lines 1-9), Algorithm 3 first takes a stream node $s$ from $V_{s}$, next finds all its adjacent stream nodes with reuse edges, then finds all adjacent stream nodes with reuse edges for the found stream nodes, and finally recursively finds the $s u b-R G$ including $s$. The function findGenerator, finding the generator for a $s u b-R G$, initially lets the first stream node in $s u b-R G$ be the generator of the $s u b-R G$, and then searches the $s u b-R G$ for a different stream node that is the reuse source of the generator. If present, it lets the found stream node be the new generator and goes on searching; if not, it returns the generator.

The SRG in Figure 4(a), for example, has three sub-RGs: $o_{0}$ composes $s u b$ $R G_{0}$ and $g_{0}$ is $o_{0} ; s_{3}$ composes $s u b-R G_{1}$; and $g_{1}$ is $s_{3} ; s_{0}, s_{1}$ and $s_{2}$ compose 


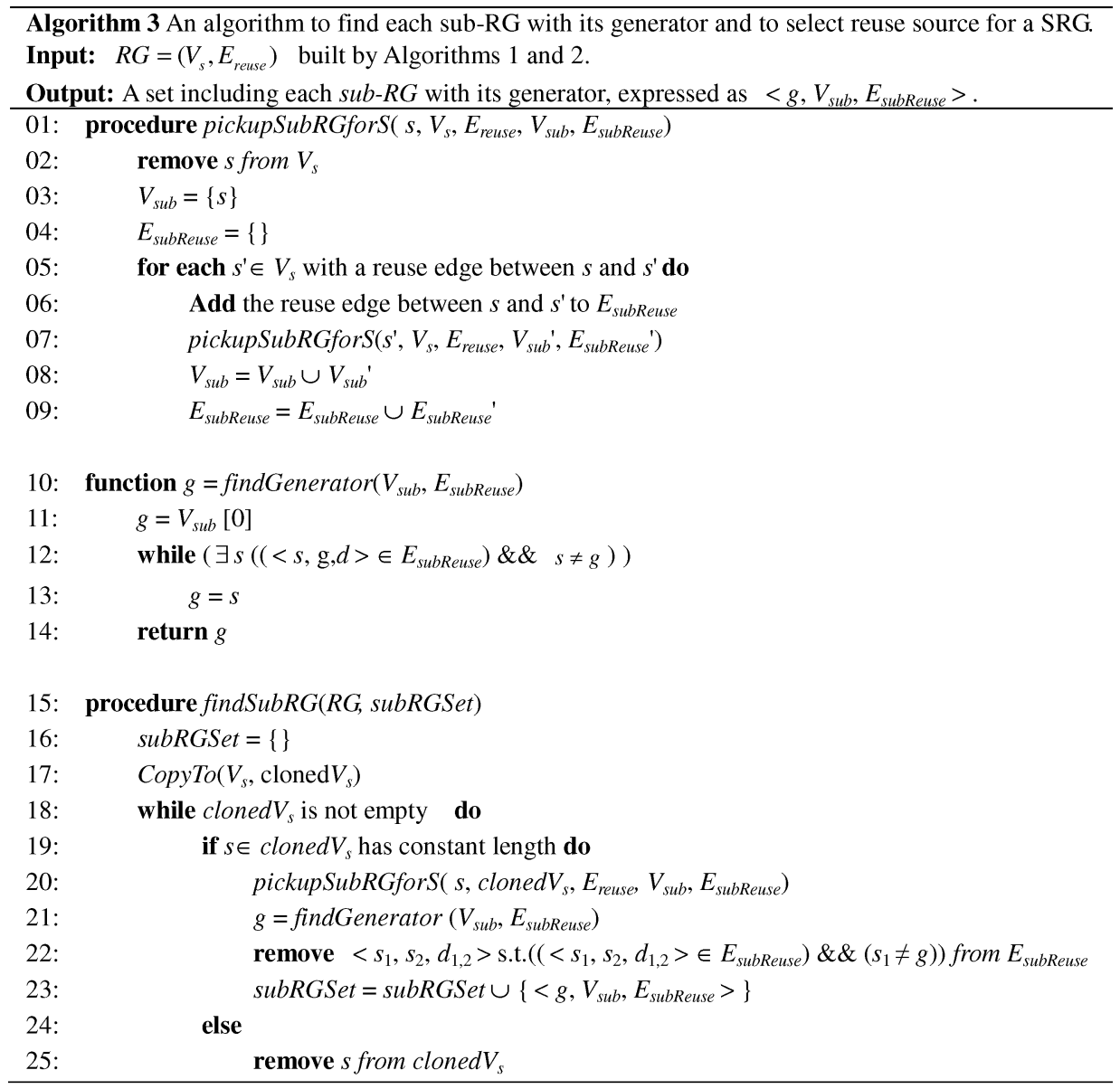

Fig. 11. Algorithm finding each $s u b-R G$ and to select the reuse source(s) for a SRG.

sub-RG $R G_{2}$ and $g_{2}$ is $s_{2}$. In $s u b-R G_{2}$, the reuse edge from $s_{1}$ to $s_{0}$ is cut. The optimized SRG is shown in Figure 4(b).

\subsection{Expanding the Optimized SRG for Partial Reuse}

We expand the optimized SRG to make it also depict partial reuse. To quantify the partial reuse, we give the following definition.

Definition 1. The supersequence of the reuse source and destination pair is the union sequence that includes the sequence accessed by the reuse source when it generates data and the sequence accessed by the reuse destination when it reuses data. For $\left\langle s_{0}, s_{1}, d_{0,1}\right\rangle(d>0)$, let $s e q_{0}$ be the sequence accessed by $s_{0}$ on iteration $i_{n}$ and $s e q_{1}$ be the sequence accessed by $s_{1}$ on iteration $i_{n}+d_{0,1}$. Their supersequence on iteration $i_{n}$ is the minimum union sequence of $s e q_{0}$ and $s e q_{1}$. Figure 12 graphically draws their supersequence. For $s_{1}$ and $s_{3}$ in Figure 2, 


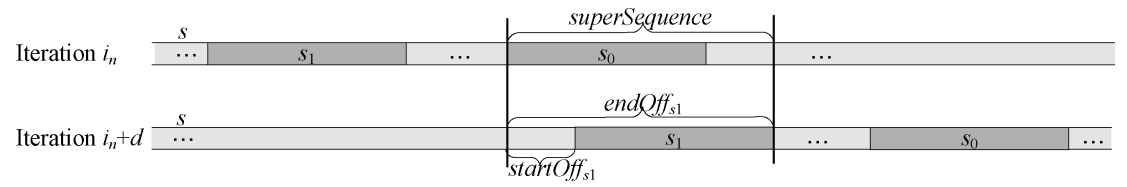

Fig. 12. Supersequence, start, and end offsets of $s_{0}$ and $s_{1}$ s.t. partial reuse exists between them.

their supersequence on iteration $i_{1}$ is $g=\langle s,(i 1+2) * N,(i 1+3) * N, 1\rangle$, as shown in Figure 5.

As discussed before, the stream compiler allocates a buffer for the supersequence of stream references with partial reuse, and uses the SDR to specify which part of the common buffer is used by each stream reference. Therefore, we must define which part of the supersequence is accessed by the reuse source or destination when it generates or reuses data. We give the following definitions:

Definition 2. The start offset, denoted as startOff, of the reuse source (or destination) is the offset from the start of its supersequence to the start of the sequence accessed when it generates (or reuses) data.

Definition 3. The end offset, denoted as endOff, of the reuse source (or destination) is the offset from the start of its supersequence to the end of the sequence accessed when it generates (or reuses) data.

Figure 12 graphically draws the start and end offsets of $s_{1}$. Therefore, the sequence accessed by $s_{1}$ when it reuses data, that is, on iteration $i_{n}+d$, can be expressed as $\left\langle\right.$ supersequence, start $O f_{s 1}$, endOff si $_{1}$, stride $\rangle$, and the sequence accessed by $s_{1}$ on iteration $i_{n}$ can be got by shifting that sequence backward $d$ iterations. For the example in Figure 2, the sequence accessed by $s_{0}$ on iteration $i_{1}+2$ can be expressed as $\langle g, 0, N, 1\rangle$; the sequence accessed by $s_{3}$ on iteration $i_{1}$ can be expressed as $\langle g, 0, N / 2,1\rangle$, as shown in Figure 5 .

Based on Lemma 4, we reach the conclusion that for two generators $g_{0}$ and $g_{1}$ with super $_{0,1}$ as their supersequence, if partial location reuse $\left\langle g_{0}, g_{1}, d_{0,1}\right\rangle\left(d_{0,1} \geq\right.$ $0)$ is satisfied, the stream node $s_{0, k}$ such that $\left\langle g_{0}, s_{0, k}, d_{0, k}\right\rangle$ is satisfied, accesses partial locations that are accessed by super $_{0,1}$ before $d_{0, k}$ iterations, while the stream node $s_{1, k}$, such that $\left\langle g_{1}, s_{1, k}, d_{1, k}\right\rangle$ is satisfied, accesses partial locations that are accessed by super ${ }_{0,1}$ before $d_{0,1}+d_{1, k}$ iterations. This means partial location reuse exists between super $_{0,1}$ and all stream nodes in $s u b-R G_{0}$ and sub-RG $G_{1}$, with super ${ }_{0,1}$ as their reuse source.

Next, we give the condition that should be satisfied if the partial value reuse exists between super $_{0,1}$ and all stream nodes in $s u b-R G_{0}$ and $s u b-R G_{1}$ :

Theorem 2. For two generators $g_{0}$ and $g_{1}$ such that partial location reuse $\left\langle g_{0}, g_{1}, d_{0,1}\right\rangle\left(d_{0,1} \geq 0\right)$ is satisfied, with super ${ }_{0,1}$ as their supersequence, if super $r_{0,1}$ remains unchanged in the next $d_{0,1}$ iterations, the partial value reuse exists

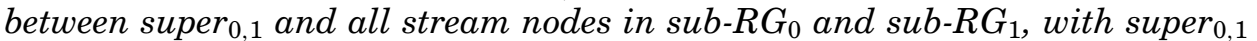
as their reuse source. 


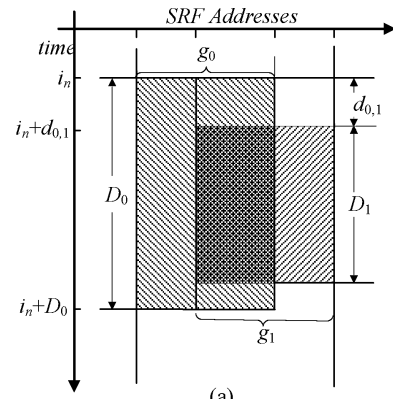

(a)

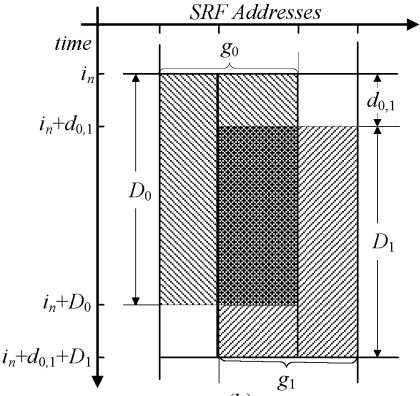

(b)

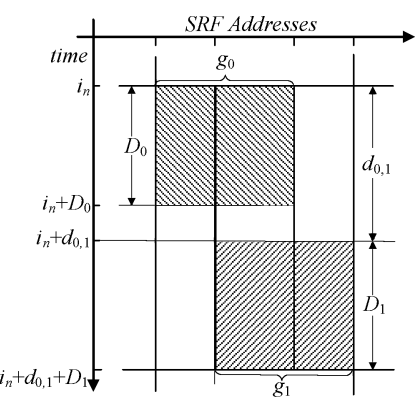

Fig. 13. Which parts of super $_{0,1}$ reused as stream nodes in $s u b-R G_{0}$ and $s u b-R G_{1}$ at what iteration.

Proof. Let $D_{0}$ be the maximum reuse distance in $s u b-R G_{0}$, and $D_{1}$ be the maximum reuse distance in $s u b-R G_{1}$. Figure 13 describes which parts of super $_{0,1}$ are reused by the stream nodes in $s u b-R G_{0}$ and $s u b-R G_{1}$ at what iteration. Values generated by $g_{0}$ are unchanged from iteration $i_{n}$ to $i_{n}+D_{0}$ due to the value reuse in $s u b-R G_{0}$, so this part of super ${ }_{0,1}$ is not changed from iteration $i_{n}$ to $i_{n}+D_{0}$, so partial value reuse exists between super $r_{0,1}$ and the stream nodes in $s u b-R G_{0}$. Similarly, values generated by $g_{1}$ are unchanged from iteration $i_{n}+d_{0,1}$ to $i_{n}+d_{0,1}+D_{1}$. Because super ${ }_{0,1}$ remains unchanged in next $d_{0,1}$ iterations, this part of super ${ }_{0,1}$ is not changed from iteration $i_{n}$ to $i_{n}+d_{0,1}+D_{1}$, so partial value reuse exists between super $_{0,1}$ and the stream nodes in $s u b-R G_{1}$.

Therefore, $s u b-R G_{0}$ and $s u b-R G_{1}$ such that their generators $g_{0}$ and $g_{1}$ satisfy Theorem 2 can be merged into one new $s u b-R G$, with super $_{0,1}$ as its new generator. The stream nodes in the merged $s u b-R G$, that is, stream nodes in sub-RG $G_{0}$ and $s u b-R G_{1}$, reuse the whole or partial data generated by super $_{0,1}$ before some iterations. Based on Definition 2 , the sequence accessed by super $_{0,1}$ on iteration $i_{n}$ is the union sequence of the sequence accessed by $g_{0}$ on iteration $i_{n}$, that is, $\left\langle s\right.$, start $_{0, i_{n}}, e n d_{0, i_{n}}$, stride $\rangle$, and that by $g_{1}$ on iteration $i_{n}+d$, that is, $\left\langle s\right.$, start $_{1, i_{n}}+d \times v_{n}$, end $_{1, i_{n}}+d \times v_{n}$, stride $\rangle$. Therefore, the start expression of super $_{0,1}$ can be expressed as startSuper $i_{n}=\min \left\{\right.$ start $_{0, i_{n}}$, start $\left._{1, i_{n}}+d \times v_{n}\right\}$. According to Lemma 1 , we get $d=0$ if $v_{n}=0$ or $d=c s_{0} / v_{n}-c s_{1} / v_{n}$ if $v_{n} \neq 0$, so startSuper $_{i_{n}}=$ start $_{0, i_{n}}-\left(c s_{0}-c s_{0} / C \times v_{n}\right)+\min \left\{c s_{0}-c s_{0} / C \times v_{n}, c s_{1}-c s_{1} / C \times v_{n}\right\}$. Similarly, the end expression of super $_{0,1}$ can be expressed as endSuper ${ }_{i_{n}}=$ end $_{0, i_{n}}-\left(c e_{0}-c s_{0} / C \times v_{n}+\max \left\{c e_{0}-c s_{0} / C \times v_{n}, c e_{1}-c s_{1} / C \times v_{n}\right\}\right.$. From Figure 13, we can intuitively see the relationship between super $_{0,1}$ and $g_{0}, g_{1}$. This result can be expanded straightforwardly for multiple generators with partial reuse, and is summarized in the following text.

Theorem 3. For $m$ generators, $g_{0}, \cdots, g_{m-1}$, such that partial reuse exists between them and $g_{k}(0 \leq k<m)$ is their new generator, the start expression of their supersequence is starth $_{i_{n}}-\left(c s_{k}-c s_{k} / C \times v_{n}\right)+\min \left\{c s_{j}-c s_{j} / C \times v_{n}(0 \leq\right.$ $j<m)$, and the end expression of their supersequence is end $k_{i_{n}}-\left(c e_{k}-c s_{k} / C \times\right.$ $\left.v_{n}\right)+\max \left\{c e_{j}-c s_{j} / C \times v_{n}(0 \leq j<m)\right\}$. 
The part of the new generator that is reused by each old generator should be determined. For the new generator $g_{\text {new }}$ and an old generator $g_{0}$ such that $\left\langle g_{\text {new }}, g_{0}, d_{0}\right\rangle$ is satisfied, let start $_{0, i_{n}+d_{0}}$ and end $_{0, i_{n}+d_{0}}$ be the start and end expressions of the sequence accessed by $g_{0}$ after $d_{0}$ iterations, and start $_{n e w, i_{n}}$ be the start of the sequence accessed by $g_{\text {new }}$ on the current iteration. The start and end offsets of $g_{0}$ can be expressed as startOff $0=$ start $_{0, i_{n}+d_{0}}-$ start $_{\text {new }, i_{n}}$ and endOff $0=e n d_{0, i_{n}+d_{0}}-$ start $_{\text {new, } i_{n}}$. According to the result of Theorem 3, we get that startOff $0=\left(c s_{0}-c s_{0} / C \times v_{n}\right)-\left(c s_{n e w}-c s_{n e w} / C \times v_{n}\right)$; endOff $0=$ $\left(c e_{0}-c s_{0} / C \times v_{n}\right)-\left(c s_{n e w}-c s_{n e w} / C \times v_{n}\right)$. This result is summarized in the following text.

Theorem 4. For $m$ generators, $g_{0}, \cdots, g_{m-1}$, such that partial reuse exists between them, the start and end offsets of $g_{k}(0 \leq k<m)$ can be expressed as ${\text { start } O f f_{k}=\left(c s_{k}-c s_{k} / C \times v_{n}\right)-\min \left\{c s_{j}-c s_{j} / C \times v_{n}(0 \leq j<m)\right\} \text { and endOff }}_{k}=\left(c e_{k}-\right.$ $\left.c s_{k} / C \times v_{n}\right)-\min \left\{c s_{j}-c s_{j} / C \times v_{n}(0 \leq j<m)\right\}$.

When merging $s u b-R G_{0}$ and $s u b-R G_{1}$, we first insert a new stream node for super $_{0,1}$, then add new reuse edges from super ${ }_{0,1}$ to $g_{0}$ with the value equal to 0 and from super $_{0,1}$ to $g_{1}$ with the value equal to $d_{0,1}$, and finally update the

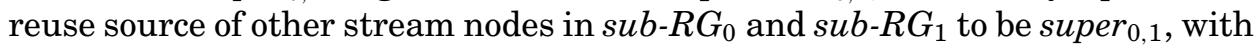
new values. Similar treatment is done to merge multiple $s u b-R G s$ with partial reuse existing among their generators.

Algorithm 4 in Figure 14 expands the SRG for partial reuse. First, it identifies all sub-RGs where partial reuse exists among their generators and determines the new generator as follows (lines 4-13): (i) Take $s u b-R G_{0}$ into a set, called mergedSet, used to hold the $s u b-R G s$ that can be merged into one new $s u b-R G$, and let $s u b-R G_{0}$ 's generator be the new generator, $g_{n e w}$, of the merged $s u b-R G$ (lines 4-7). (ii) Find another generator, $g^{\prime}$, which has partial value reuse relation with $g_{\text {new }}$, place it into the mergedSet, and update the new generator as their supersequence (lines 9-13), according to Theorem 3. If partial reuse exists between two generators on the same iteration, the stream node of a earlier called kernel is the reuse source. Step (iii) repeats (ii) until there is no stream node having partial value reuse with $g_{n e w}$. Second, it merges all sub-RGs in the mergedSet into a new $s u b-R G$ by building a new stream node for $g_{\text {new }}$, inserting a reuse edge, with $d_{i}$ as its value, from $g_{\text {new }}$ to the old generator $g_{i}\left(g_{i} \in\right.$ mergedSet) such that $\left\langle g_{n e w}, g_{i}, d_{i}\right\rangle$ is satisfied, and updating the reuse source of $s_{i, k}$ to be $g_{n e w}$ and its reuse distance to be $d_{i}+d_{i, k}$ for each stream node $s_{i, k}$ such that $\left\langle g_{i}, s_{i, k}, d_{i, k}\right\rangle$ (lines 15-23). Finally, Algorithm 4 repeats the previously mentioned steps until all partial reuse is described in the SRG.

Thus, all sub-RGs in a mergedSet are merged into one new sub-RG. Following expansion, all stream nodes are divided into $p$ connected $s u b-R G s$ and reuse the whole or partial values generated by their generator before some iterations. Figure 4(c), for example, is expanded from the optimized SRG in Figure 4(b) for the code from Figure 2. Partial value reuse exists between the generators $s_{3}$ and $s_{2}$, so $s u b-R G_{1}$ and $s u b-R G_{2}$ are merged into one new $s u b-R G$ (called $\left.s u b-R G_{1}^{\prime}\right)$. The new generator is the stream reference $\langle s,(i+2) \times N,(i+3) \times N, 1\rangle$ and a stream node $g$ is built for it. The reuse sources of $s_{3}, s_{2}, s_{1}$, and $s_{0}$ are updated to be $g$. 


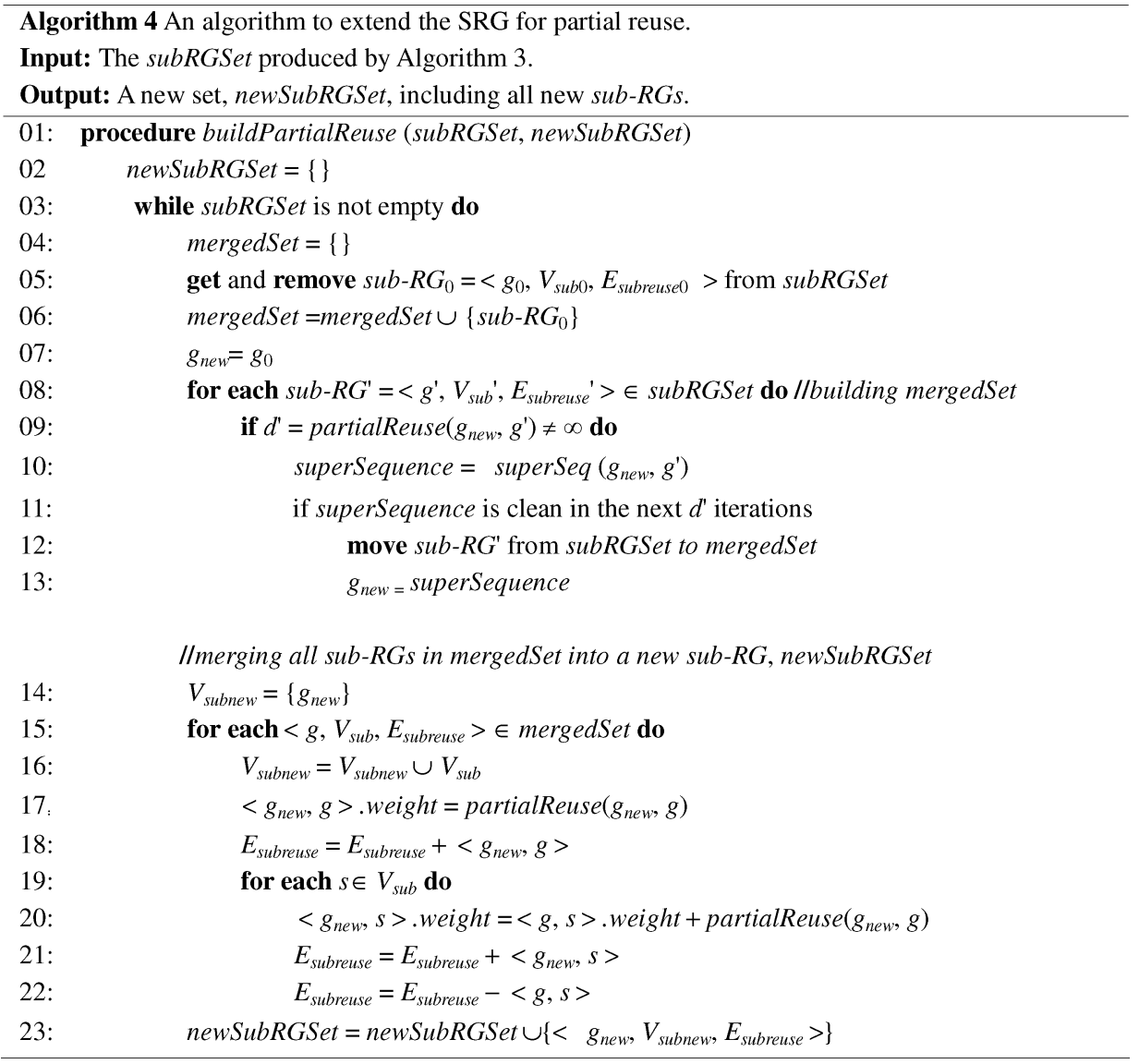

Fig. 14. Algorithm extending the SRG for partial reuse.

\section{CONSTANT-BOUND STREAM REPLACEMENT}

In this section, we transform a stream-level program to make use of the reuse supplied by loop-dependent stream references, based on the built and optimized SRG. The procedure consists of five steps, as follows:

Determining the number of constant-bound stream references. We calculate the number of constant-bound streams, $N_{i}$, needed to hold the data generated by the generator, $g_{i}$. To eliminate the load of the reuse destinations in $s u b$ $R G_{i}, D_{i}+1$ constant-bound streams are needed [Callahan et al. 1990], thus providing $D_{i}$ constant-bound stream references for values computed on the $D_{i}$ previous iterations plus one for the values computed on the current iteration.

Determining the loop unrolling factors. To eliminate all SRF-to-SRF moves that implement data permutation among $N_{i}$ stream references in $s u b-R G_{i}$, the loop should be unrolled at least $N_{i}$ times. Therefore, to eliminate all data permutation for all sub-RGs, the unrolling factors, $N U$, equals $\max \left\{N_{0}, \ldots, N_{p-1}\right\}$. 
This value may be large, which means a large SRF space requirement for all identified reuse. However, due to the limited SRF capacity, the built SRG will be pruned as described in Section 7, which limits the value.

Reference replacement. This procedure first creates $N_{i}$ constant-bound streams, $g_{i}^{0}, \ldots, g_{i}^{N_{i}-1}$ for each generator, $g_{i}$. Next, the innermost loop body is unrolled $N U$ times and a statement $i_{n}=i_{n}+1$ is inserted just before each unrolled loop body except the first one. Finally, the procedure replaces the references involved by references to corresponding constant-bound stream references and inserts stream loads or saves in the right place.

For each reference $s_{i, j}$ such that $\left\langle g_{i}, s_{i, j}, d_{i, j}\right\rangle$ is satisfied, in order to specify which part of data generated by $g_{i}$ is reused by $s_{i, j}$ after $d_{i, j}$ iterations, the start and end offsets of $s_{i, j}$, denoted startOff $f_{i, j}$ and endOff $f_{i, j}$, are computed according to Theorem 4.

In the first unrolled body, the references to $g_{i}$ are replaced with $g_{i}^{0}$; the references to $s_{i, j}$ are replaced with the stream reference $\left\langle g_{i}^{d_{i, j}}\right.$,

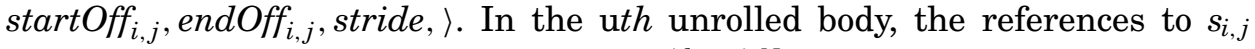
are replaced with the stream reference $\left\langle g_{i}^{\left(d_{i, j}-u\right) \mid N_{i}},{\text { start } O f f_{i, j}}_{\text {, endOff }}\right.$, stride $\rangle$.

For the references to the generator $g_{i}$, if they are produced by their first kernel call, the produced constant-bound streams are saved back just after its first kernel call. If they are loaded by their first kernel calls, a load of $g_{i}$ is inserted just before the first kernel call involving the whole or part of $g_{i}$. If $g_{i}$ is loop-invariant (i.e., $v_{n}=0$ ), all loads or saves are merged into a single load or save and moved just before or after the innermost loop body.

Initialization. This procedure initializes the constant-bound streams, processes the innermost loop index variable, and produces the epilogue loop. We do not detail the last two parts of the procedure, as they are straightforward.

For $s u b-R G_{i}, D_{i}$ constant-bound streams should be initialized with the right values. For the stream node $s_{i, j}$ such that $\left\langle g_{i}, s_{i, j}, d_{i, j}\right\rangle$, data used by $s_{i, j}$ on the first iteration should be assigned to $g_{i}^{d_{i, j}}$. If multiple stream nodes have the same reuse distance, their supersequence is assigned. If there is no reuse distance equal to $d_{i, j}+1$, data used by $s_{i, j}$ in the next iteration is assigned to $g_{i}^{d_{i, j}+1}$, and so on.

By now, the reuse supplied by loop-dependent stream references has been exploited and corresponding stream loads have been eliminated. Figure 5(c) shows the final code of the example in Figure 2. There are two sub-RGs, sub$R G_{0}=\left\{o_{0}\right\}$ and $s u b-R G_{1}=\left\{s_{0}, s_{1}, s_{2}, s_{3}\right\} ; g_{0}=o_{0}$ and $g_{1}=\langle s,(i+2) \times N,(i+$ $3) \times N, 1\rangle$. In $s u b-R G_{1}, s_{3}$ reuses part of the generator $g$ on the same iteration. Its start and end offsets equal 0 and $N / 2$, respectively. Other stream references reuse the whole of $g$. The loop should be unrolled three times to eliminate SRF-to-SRF data moves among four constant-bound streams in sub-RG $G_{1}$.

\section{REDUCING SRF PRESSURE}

The exploitation of stream reuse, especially for the loop-carried reuse, requires extending the live ranges of reuse sources, which may increase the SRF 
Table I. Symbols Used in Our Greedy Algorithm

\begin{tabular}{|c|c|}
\hline Symbol & Definition \\
\hline$\delta\left(S_{i, j}\right)$ & Difference of the stream node $S_{i, j}$ 's reuse distance and the next less reuse \\
& distance in $s u b-R G_{i}$ \\
$\operatorname{SFR}\left(g_{i}\right)$ & \\
$M E M\left(S_{i, j}\right)$ & \\
$C_{r}$ & \\
\hline
\end{tabular}

pressure. If the SRF requirement at any time is beyond the SRF capacity, the stream compiler [Mattson 2002] will spill the least recently used stream, which fails to fully exploit stream reuse and to reduce memory accesses. Therefore, we aid the stream compiler to choose the stream reuse to be exploited such that as many memory accesses are eliminated by the stream reuse exploitation as possible within the limited SRF capacity.

If the SRF space requirement is beyond the SRF capacity, we cut some reuse edges in the SRG to limit the requirement to less than the SRF capacity. Our goal is to choose the reuse edges to be cut such that as many memory accesses are eliminated by the stream reuse exploitation as possible within the limited SRF capacity. This can be achieved by modeling the task as a knapsack problem. In the model, the SRF capacity is the knapsack weight. The boxes to be put in the knapsack are the stream nodes. The value of the box is the number of memory accesses eliminated by its reuse exploitation, which equals that of memory accesses required to load the stream node. The weight of the box is the space requirement to exploit its reuse. For the stream node $s_{i, j}$ in $s u b-R G_{i}$ with $d_{i, j}$ as its reuse distance, let $s_{i, j-1}$ be the stream node having the next less reuse distance, $d_{i, j-1}$. To exploit the reuse supplied by $s_{i, j-1}$, the data generated by $g_{i}$ on $d_{i, j-1}$ previous iterations plus the current iteration has been held in the SRF. Therefore, in order to exploit the reuse supplied by $s_{i, j}$, it takes extra SRF buffers to hold the data generated by $g_{i}$ from previous $d_{i, j-1}+1$ to previous $d_{i, j}$ iterations. Hence, we use the total size of these extra SRF buffers as $s_{i, j}$ 's weight. For the knapsack problem, we should maximize the values brought by the boxes carried in the knapsack.

We use a greedy approximation algorithm to find a solution. We use the symbols described in Table I. In this approximation scheme, cutting edges is based on the ratio of benefit to cost, or in this case, the ratio of saved memory transfers to the SRF space requirement, computed with Equation (1). Sub$R G_{i}$ requires $S R F\left(g_{i}\right) \times N_{i}$ words of the $\mathrm{SRF}$, with $N_{i}-1$ of them holding the data generated by $g_{i}$ on the $N_{i}-1$ previous iterations plus one on the current iteration. Therefore, the total SRF space requirement of loop $L$ is $\sum_{0 \leq i<p} g_{i} \times N_{i}$, without considering to coalesce streams whose live ranges did not interfere. Therefore, we get initial $C_{r}$ with Equation (2). The candidates to be cut must be ones with the largest reuse distance in each sub-RG [Mattson 2002]. Our greedy algorithm first puts all boxes in the knapsack and tests whether the knapsack is overweight, or in this case, $C_{r}<0$. If so, it chooses to cut the one with minimum ratio among the candidates, uses Equation (2) to update the $C_{r}$, and tests whether there is remaining $\mathrm{SRF}$ space, that is, $C_{r}>0$. If so, our greedy algorithm is finished; otherwise, it updates the candidate set 


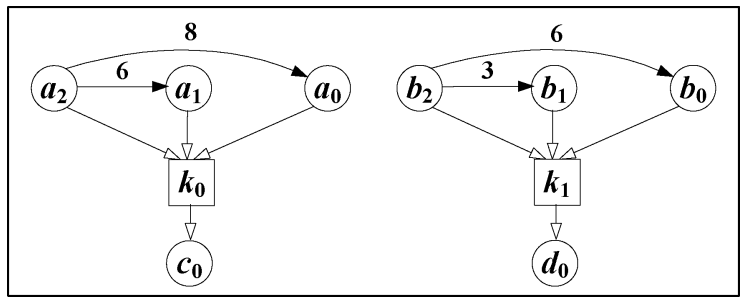

Fig. 15. Example SRG for the greedy algorithm evaluation.

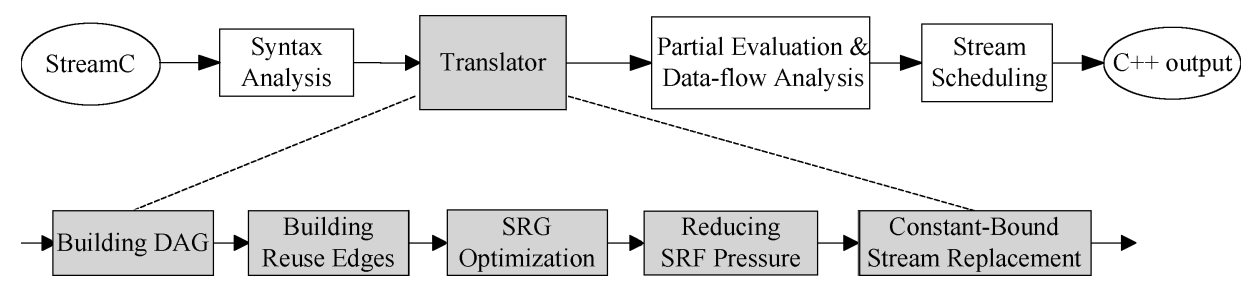

Fig. 16. Flowchart of our scheme.

and repeats cutting and testing. The greedy algorithm's total running time is $n \log n$ where $n$ is the number of the reuse destinations, which is independent of stream processor architecture.

$$
\begin{gathered}
\text { Ratio }=\frac{\operatorname{Mem}\left(s_{i, j}\right)}{\delta\left(s_{i, j}\right) \times S R F\left(g_{i}\right)} \\
C_{r}=C_{S R F}-\sum_{i=0}^{p-1} S R F\left(g_{i}\right) \times N_{i}
\end{gathered}
$$

Unfortunately, the greedy approximation can produce suboptimal results. To see this, consider the SRG in Figure 15, with assumption that all stream nodes in the SRG have the same length, $N$, and the same record type, which means $a_{2}$ and $b_{2}$ take the same SRF space, and the benefits of exploiting the reuse supplied by each stream node are the same, assuming that we have a stream processor with the $\mathrm{SRF}$ capacity equal to $13 \times N$. The greedy algorithm would first cut reuse edges from $b_{2}$ to $b_{0}$, then from $b_{2}$ to $b_{1}$, and finally from $a_{2}$ to $a_{0}$. This would result in the elimination of $N$ memory accesses with the $\mathrm{SRF}$ requirement equal to $13 \times N$. However, $2 \times N$ memory accesses can be eliminated by first cutting reuse edges from $a_{2}$ to $a_{0}$ and then from $a_{2}$ to $a_{1}$ with the $\mathrm{SRF}$ requirement equal to $12 \times N$.

\section{EXPERIMENT}

We have implemented a translator, shown in Figure 16, indicated by dark shading, based on the stream compiler developed by Stanford University [Das et al. 2006; Mattson 2002]. Figure 16 shows the flowchart of our scheme. The 
Table II. Benchmark

\begin{tabular}{|l|l|c|c|}
\hline Benchmark & Source & Prob. Size & Max Comp./Mem. \\
\hline Laplace & NCSA & $1 K \times 1 K$ & 2.5 \\
Swim & Spec2000 & $512 \times 512$ & 3.67 \\
MG & NPB & $128 \times 128 \times 128$ & 3.67 \\
GEMM & BLAS & $512 \times 512$ & 341 \\
FFT & HPCC & $4 K$ & 44 \\
Jacobi & - & $256 \times 256$ & 4.98 \\
QMRCGSTAB & - & $800 \times 800$ & 1.85 \\
MVM & - & $832 \times 832$ & 1.5 \\
Test1 & - & $1 K \times 1 K$ & 1 \\
Test2 & - & $1 K \times 1 K$ & 2 \\
\hline
\end{tabular}

translator takes an (abstract syntax tree) (AST) of a StreamC program as its input, builds the SRG for each loop body, unrolls each loop body and replaces loop-dependent stream references by constant-bound stream references. To our best knowledge, the Stanford Imagine and Merrimac team represents the best existing techniques for the compilation of stream processors with large onchip stream buffering, or the SRF. They published work for improving locality by exploiting reuse in Mattson [2002]. Our work is based on the results and exploits the reuse supplied by streams with unknown bounds and stream reuse over loop iterations. Readers can refer to Das et al. [2006] and Mattson [2002] to know the function of the other modules of the compiler.

Table II summarizes the 10 benchmarks we used. Test 1 and Test 2 represent the examples in Figure 2 and Figure 9, respectively. Except QMRCGSTAB, nine of them are scientific kernels, with each having only one loop in the streamlevel program. MVM calculates the multiplication of two band matrices. QMRCGSTAB [Chan et al. 1994] is a real application and used to solve large sparse linear systems with asymmetrical coefficient matrices with the Krylov subspace iteration method.

The column "Max Comp./Mem." of a benchmark is quantified with the total number of the computations of an application divided by its minimum data transfers, with all loads from the same location counted as once data transfer and all stores to the same location counted as once data transfer. The "Max Comp./Mem." of a benchmark is gained only if its all redundant loads and stores are eliminated. This item is used to evaluate the reuse exploitation degree of our scheme later.

The code optimized with and without our method is performed on a cycleaccurate simulator of the Imagine stream processor, with a 128KB SRF.

We first demonstrate the importance of our exploitation of stream reuse on the nine scientific kernels in Section 8.1, then on the complete application QMRCGSTAB in Section 8.2, and finally the effectiveness of reducing the SRF pressure in Section 8.3.

\subsection{Scientific Kernels}

Table III shows the effectiveness of our reuse exploitation on the nine scientific kernels. The row Stream references illustrates the number of loop-dependent stream references of each loop body. The stream compiler generates a stream 


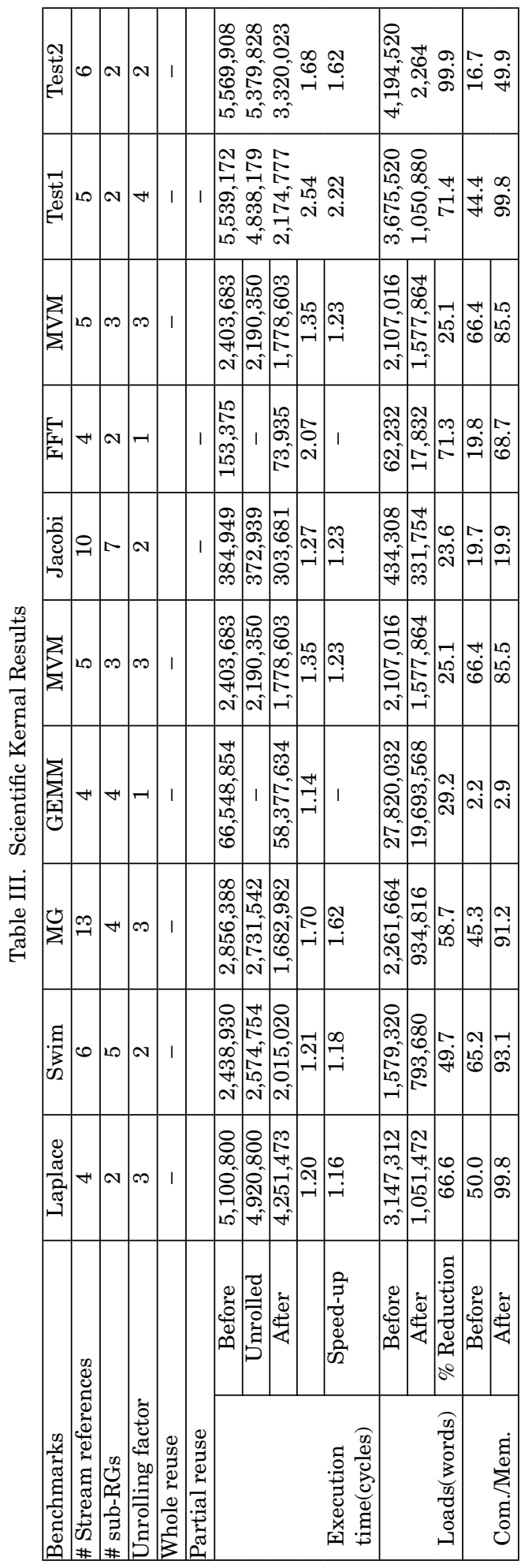

ACM Transactions on Architecture and Code Optimization, Vol. 7, No. 2, Article 11, Pub. date: September 2010. 


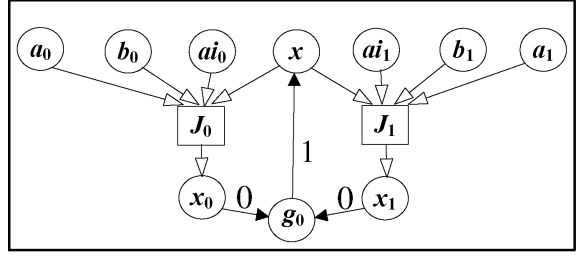

(a) Jacobi

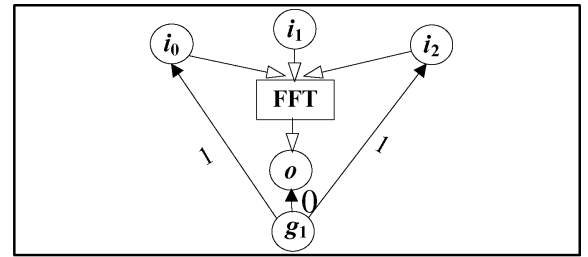

(b) FFT

Fig. 17. SRGs of Jacobi(a) and FFT(b).

load or store for each such reference before our transformation. The row subRGs describes the number of sub-RGs in each SRG. A stream load (or save) is needed only for each generator of each $s u b-R G$ on each iteration or just before (or after) the innermost loop body. These two rows also somewhat reflect the complexity of building the SRG. The row Unrolling factor shows the number of times that each loop is unrolled to avoid SRF-to-SRF data moves.

The rows "Whole reuse" and "Partial reuse" show whether a benchmark contains the opportunities for the whole or partial reuse. Seven scientific kernels, Laplace, Swim, MG, GEMM, MVM, Test 1, and Test 2, contain the whole reuse; three scientific kernels, Jacobi, FFT, and Test 1, contain partial reuse. Figure 17 draws the SRGs of Jacobi and FFT. The outputs, $x_{0}$ and $x_{1}$, of kernels $J_{0}$ and $J_{1}$ are concatenated together to be an input $x$ of the next iteration, which indicates the generator $g_{0}$ is the union of $x_{0}$ and $x_{1}$. For FFT, the first half and second half of its output $o$ act as two inputs, $i_{0}$ and $i_{2}$, in the next iteration. Therefore, the generator $g_{1}$ accesses the same sequence with the stream reference $o$; the stream references $i_{0}$ and $i_{1}$ access the first and second half parts of data used by $g_{1}$ on last iteration, respectively.

The row Execution time demonstrates the significant effect of our automatic method on the execution time. The row Unrolled shows the speed of the original loop after unrolling it an amount equivalent to that done by our method. This row shows the impact of unrolling on the performance. The speed-up column has two fields. The first field shows the speed-up of the transformed code over original code; our method gains from 1.14 to 2.54 times speed-up. The second field, if present, shows the speed-up of the transformed code over the unrolled code; our method gains from 1.16 to 2.22 times speed-up.

As the main contribution of our method is the elimination of the memory loads, the row Loads illustrates this ability. The row \% Reduction equals (BeforeAfter)/Before $\times 100 \%$ showing the percentage of the reduced loads from the loads in original code. GEMM demonstrates interesting results. It has much loopinvariant reuse. Except the initial loads, one out of the three input streams does not need any load in the innermost loop. Test 2 has a similar improvement. All loads within an iteration are eliminated. As our method aims to only reduce stream loads, there are still redundant stream saves that can be optimized in Test 2. For Laplace, an application with intensive memory accesses, its stream loads are reduced to nearly one third. As a result, the memory-intensive application is no longer limited by memory accesses, and its computations take 
a longer time than its memory accesses after our optimization. Our method eliminates two loads on each iteration of FFT and one load on each iteration of Jacobi. FFT benefits greatly from our method, although it is a computationintensive application. Without our method, its stream program is somewhat limited by memory accesses because its intensive computations are accelerated by plenty of ALUs of the stream processor. Our method eliminates $71.3 \%$ of its memory loads, and it becomes limited by computations after our optimization. For Test 1, three loads, two supplied by whole reuse and one by partial reuse, on each iteration are eliminated. Thus, $71.3 \%$ of its memory loads are not necessary any more. For the rest applications, the memory accesses brought about by 1 out of 2, 2 out of 3 and 6 out of 13 stream references in Swim, MVM, and MG are eliminated, respectively. The experimental results show that the performance improvement is nearly proportional to the reduction of memory transfers for all benchmarks.

The row \% Comp./Mem. evaluates the exploitation degree of the stream reuse and is quantified by comparing the achieved Comp./Mem. to the maximum Comp./Mem. in Table II. If its value equals 100\%, all reuse is exploited. Our automatic method effects all stream reuse in Laplace, Swim, MG, MVM, and Test1, although they do not get the maximum Comp./Mem.: The load of kernel code introduces extra memory transfers and thus reduces the Comp./Mem. in Laplace and Test 1; some redundant data is introduced in Swim, MG, and MVM when mapping them on the stream processor in order to utilize the architecture features [Zhang et al. 2008]. For Test 2, the stream reuse is completely exploited, though there are still redundant stream saves which are not considered by our method, so only $49.9 \%$ maximum Comp./Mem. is gained. As GEMM is optimized with loop-tiling when it is mapped on the stream processor, some reuse does not exist in the innermost loop, and only $2.9 \%$ maximum Comp./Mem. is gained. However, our method exploits all its reuse with respect to the innermost loop. Although Jacobi has an inherent Comp./Mem. of 4.98, most reuse exists in the outer loop. Therefore, its reuse cannot completely be captured. Our automatic method only gains $19.9 \%$ maximum Comp./Mem.. However, our method exploits all its stream reuse with respect to the innermost loop. Some reuse of FFT does not exist in the innermost loop because FFT is mapped on to the stream processor after a strip-mining optimization [Das et al. 2006], so our approach gains only $68.7 \%$ maximum Comp./Mem., which is much higher than that before optimization.

\subsection{Complete Application}

We performed the test on the complete application, QMRCGSTAB [Chan et al. 1994]. It consists of four 2-level loops. Two of them, called QMR iteration, perform the main calculations. We only perform each outermost loop once. Figure 18 shows the SRG of the first QMR iteration. As shown, the reuse relationship among stream references is complicated. Table IV summarizes the characteristics of the SRG of each loop of QMRCGSTAB. It has 43 loopdependent stream references in total. Each loop requires distinct unrolling factors. This further demonstrates the complex reuse relationship in a real 


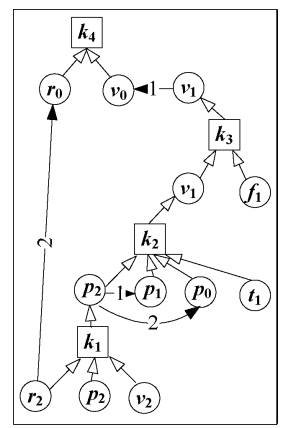

Fig. 18. SRG of the first QMR loop.

Table IV. Characteristics of SRGs

\begin{tabular}{|l|l|l|l|l|}
\hline Loop No. & 1 & 2 & 3 & 4 \\
\hline Stream references & 14 & 6 & 17 & 6 \\
sub-RGs & 8 & 5 & 9 & 5 \\
Unrolling factors & 3 & 1 & 3 & 1 \\
\hline
\end{tabular}

Table V. Effect of Stream Reuse Optimization on QMRCGSTAB's Performance

\begin{tabular}{|c|c|c|c|}
\hline & Before & After & \% Reduction \\
\hline Loads(words) & 35630663 & 24767143 & 30.5 \\
Execution time(cycles) & 36918146 & 26505621 & 28.2 \\
\% Comp./Mem. & 53 & 99.6 & - \\
\hline
\end{tabular}

application. In addition, some reuse edges in loops 1 and 3 have to be cut when the SRF requirement exceeds the SRF capacity. Therefore, it would be extremely difficult to reuse the streams manually.

Table $\mathrm{V}$ shows the results from when our method is applied to QMRCGSTAB. The application achieves a $28.2 \%$ speed-up and reduces the number of loads by $30.5 \%$. All possible stream reuse within the application has been exploited.

\subsection{Reducing the SRF Pressure}

Finally, the effectiveness of reducing the SRF pressure is demonstrated. Except QMRCGSTAB, the benefit of each reuse destination of every benchmark is larger than or equal to its cost, which means the exploitation of stream reuse does not bring extra SRF requirement, or in this case, reducing the SRF pressure brings no benefits for these benchmarks. Therefore, only QMRCGSTAB benefits from reducing the SRF pressure when its SRF requirement is beyond the SRF capacity. To test the effectiveness of our reducing the SRF pressure, we increase the problem size of this benchmark to make the SRF requirement of the transformed code beyond the SRF capacity.

Table VI demonstrates the efficiency of our approach on dealing with the SRF having insufficient capacity. The column speed-up has two fields. The first field shows the speed-up of our approach over the constant-bound stream reference replacement method without considering the SRF pressure. The second field 
Table VI. Effectiveness of the Method for Handling SRF Overflows

\begin{tabular}{|c|c|c|c|c|}
\hline \multicolumn{2}{|c|}{ Speed-up } & \multicolumn{2}{c|}{ \% Reduction of Loads } & \multirow{2}{*}{ \% Comp. Density } \\
\hline Over Without & Over Original & Over Without & Over Original & \% \\
\hline 1.07 & 1.19 & 1.12 & 1.25 & 93.2 \\
\hline
\end{tabular}

shows the speed-up of our approach over the original code. The column $\%$ Reduction of Loads also has such two fields. Without the knapsack model or greedy algorithm, 29 stream loads are needed on each iteration for the code optimized with the constant-bound stream reference replacement method. However, our greedy algorithm reduces the number to 27 and gains a 1.07 times speed-up over the method without considering the SRF pressure and a 1.19 times speedup over the original code. The greedy algorithm effectively selects the stream reuse to be exploited within limited SRF capacity.

\section{SUMMARY AND FUTURE WORK}

In this article, we have introduced the problem of stream reuse for stream processors. We have built and analyzed the methods to effect stream reuse based around our SRG. Our approach has considered whole and partial stream reuse. Based on the SRG, we proposed a source-to-source transformation, called constant-bound stream replacement, which makes the reuse supplied by loopdependent stream references useful for the stream compiler, thus reducing unnecessary memory loads. In order to eliminate as many memory accesses by the stream reuse exploitation as possible within the limited SRF capacity, we model the task as a knapsack problem and use the greedy algorithm to find a solution. Our optimization is implemented in the StreamC/KernelC compiler. Results demonstrate that our techniques can lead to a dramatic increase in performance. For 10 benchmarks, performed on the Imagine stream processor, a speed-up of 1.14 to 2.54 times was achieved.

We are currently engaged in studying the generality of these techniques by extending our implementation and applying it to a wider class of programs. This research involves the following subprojects.

-Our current work adopts a distrust strategy when examining whether the values generated by a stream resource are changed by another stream reference from the same basic stream but in different uniformly generated sets, and regards the values of the first reference as changed by the second. In fact, this strategy may be too strict in some scenarios. We are in the process of making the examination more precise.

-Only straight-line loops are handled now. We plan to extend our approach to deal with loops with conditionals.

- Reusing the data held in the LRFs is not considered in the article. We are about to exploit such reuse based on our scheme.

\section{REFERENCES}

Agarwal, V., Hrishikesh, M. S., Keckler, S. W., and Burger, D. 2000. Clock rate versus IPC: the end of the road for conventional microarchitectures. In Proceedings of the International Syposium on Computer Architecture. ACM, New York, 248-259.

ACM Transactions on Architecture and Code Optimization, Vol. 7, No. 2, Article 11, Pub. date: September 2010. 
Ahn, J. H., ERez, M., AND Dally,W. J. 2006. The design space of data-parallel memory systems. In Proceedings of the Conference on Super-Computing (SC'06). ACM, New York, 80.

Allen, J. R. 1983. Dependence analysis for subscripted variables and its application to program transformations. Ph.D. thesis, Rice University, Houston, TX.

Allen, R. And Kennedy, K. 1992. Vector register allocation. IEEE Trans. Comput. 41, 10,12901317.

AMD Inc. AMD FireStream stream processor.

Buck, I., Foley, T., Horn, D., Sugerman, J., Fatahalian, K., Houston, M., and Hanrahan, P. 2004. Brook for GPUs: Stream computing on graphics hardware. ACM Trans. Graph. 23, 3, 777-786.

Callahan, D., CARR, S., ANd KEnNedy, K. 1990. Improving register allocation for subscripted variables. In Proceedings of the Conference on Programming Language Design and Implementation. ACM, New York, 53-65.

CARR, S. ANd Kennedy, K. 1994. Scalar replacement in the presence of conditional control flow. Softw. Pract. Exper. 24, 1, 51-77.

Chan, T. F., Gallopoulos, E., Simoncini, V., Szeto, T., and Tong, C. H. 1994. A quasi-minimal residual variant of the bi-cgstab algorithm for nonsymmetric systems. SIAM J. Sci. Comput. 15, 2, 338-347.

Crow, T. 2004. Evolution of the graphical processing unit. Ph.D. thesis, University of Nevada.

Cytron, R., Ferrante, J., Rosen, B. K., Wegman, M. N., and Zadeck, F. K. $1991 . \quad$ Efficiently computing static single assignment form and the control dependence graph. ACM Trans. Program. Lang. Syst. 13, 451-490.

Dally, W. J., Labonte, F., Das, A., Hanrahan, P., Ahn, J. H., Gummaraju, J., Erez, M., Jayasena, N., Buck, I., K., T. J., AND KaPASI, U. J. 2003. Merrimac: Super-computing with streams. In Proceedings of the Conference on Super-Computing. IEEE, Los Alamitos, CA, 35.

Das, A., Dally, W. J., AND MatTson, P. 2006. Compiling for stream processing. In Proceedings of the 15th International Conference on Parallel Architectures and Compilation Techniques. ACM, New York, 33-42.

GanNon, D., Jalby, W., AND Gallivan, K. 1988. Strategies for cache and local memory management by global program transformation. J. Parall. Distrib. Comput. 5, 5, 587-616.

Horowitz, M. And Dally, W. 2004. How scaling will change processor architecture. In Proceedings of the Solid-State Circuits Conference, 2004. IEEE, Los Alamitos, CA, 132-133.

JAYASENA, N. 2005. Memory hierarchy design for stream computing. Ph.D. thesis, Stanford University.

Jayasena, N., Erez, M., Ahn, J. H., and Dally, W. J. 2004. Stream register files with indexed access. In Proceedings of the 10th International Symposium on High-Performance Computer Architecture. IEEE, Los Alamitos, CA, 60.

Kahle, J. A., Day, M. N., Hofstee, H. P., Johns, C. R., Maeurer, T. R., and Shippy, D. $2005 . \quad$ Introduction to the cell multiprocessor. IBM J. Res. Dev. 49, 4/5, 589-604.

Kapasi, U. J., Dally, W. J., Rixner, S., Owens, J. D., And Khailany, B. 2002. The Imagine stream processor. In Proceedings of the International Conference on Computer Design. IEEE, Los Alamitos, CA, 282-288.

Kapasi, U. J., Rixner, S., Dally, W. J., Khailany, B., Ahn, J. H., Mattson, P., and Owens, J. D. 2003. Programmable stream processors. Computer 36, 8, 54-62.

Labonte, F., Mattson, P., Thies, W., Buck, I., Kozyrakis, C., and Honowitz, M. 2004. The stream virtual machine. In Proceedings of the 13th International Conference on Parallel Architectures and Compilation Techniques. IEEE, Los Alamitos, CA, 267-277.

Liao, S. W., Du, Z. H., Wu, G. S., AND Lueh, G. Y. 2006. Data and computation transformations for Brook streaming applications on multiprocessors. In Proceedings of the International Symposium on Code Generation and Optimization. IEEE, Los Alamitos, CA, 196-207.

Lindholm, E., Nickolls, J., Oberman, S., And Montrym, J. 2008. Nvidia tesla: A unified graphics and computing architecture. IEEE Micro, 39-55.

Lo, R., Chow, F., Kennedy, R., Liu, S., And Tu, P. 1998. Register promotion by sparse partial redundancy elimination of loads and stores. In Proceedings of the Conference on Programming Language Design and Implementation. ACM, New York, 26-37.

Lu, J. AND CoOPER, K. D. 1997. Register promotion in C programs. In Proceedings of the Conference on Programming Language Design and Implementation. ACM, New York, 308-319.

ACM Transactions on Architecture and Code Optimization, Vol. 7, No. 2, Article 11, Pub. date: September 2010. 
Mattson, P. 2002. A programming system for the Imagine media processor. Ph.D. thesis, Computer Systems Laboratory, Stanford University.

Mattson, P., Rixner, S., Dally, W. J., Khailany, B., Ahn, J. H., Mattson, P., and Owens, J. D. 2004. Imagine Programming System Developer's Guide. http://cva.stanford.edu.

Narayanan, M., Oliker, L., Janin, A., Husbands, P., Ye, X., AND Li, S. $2002 . \quad$ Scientific kernels on VIRAM and Imagine media processors. Lawrence Berkeley National Laboratory. http://escholarship.org/uc/item/37v9j259\#page-1

Owens, J. D., Kapasi, U. J., Mattson, P., Towles, B., Serebrin, B., Rixner, S., and Dally, W. J. 2002. Media processing applications on the Imagine stream processor. In Proceedings of the International Conference on Computer Design. IEEE, Los Alamitos, CA, 295-302.

RIXner, S. 2002. Stream Processor Architecture. Kluwer Academic Publishers, The Netherlands.

SASTRY, A. V. S. AND Ju, R. D. C. 1998. A new algorithm for scalar register promotion based on SSA form. In Proceedings of the Conference on Programming Language Design and Implementation. ACM, New York, 15-25.

Seiler, L., Carmean, D., Sprangle, E., Forsyth, T., Abrash, M., Dubey, P., Junkins, S., Lake, A., Sugerman, J., CAvin, R., ET AL. 2008. Larrabee: A many-core x86 architecture for visual computing. ACM Trans Graphics 27, 3.

Stephens, R. 1997. A survey of stream processing. Acta Informatica 34, 7, 491-541.

TAHOORI, M. AND LEE, P. Mapping vector codes to stream processor Imagine. http://webcache.googleusercontent.com/search?q=cache:0d4wHF102bUJ:citeseerx.ist.psu.edu/ viewdoc/download?doi\%3D10.1.1.112.7965\%26rep\%3Drep1\%26type\%3Dpdf+TAHOORI,+M.+ AND+LEE,+P.+Mapping+vector+codes+to+stream+processor+Imagine.\&hl=en\&gl=us.

Taylor, M. B., Kim, J., Miller, J., Wentzlaff, D., Ghodrat, F., Greenwald, B., Hoffman, H., Johnson, P., LEE, J.-W., ET AL. 2002. The Raw microprocessor: A computational fabric for software circuits and general-purpose programs. IEEE Micro 22, 2, 25-35.

West, D. 2001. Introduction to Graph Theory. Prentice Hall, Upper Saddle River, NJ.

Wolf, M. E. AND LAM, M. S. 1991. A data locality optimizing algorithm. In Proceedings of the Conference on Programming Language Design and Implementation. ACM, New York, 30-44.

Wulf, W. A. AND McKee, S. A. 1995. Hitting the memory wall: Implications of the obvious. SIGARCH Comput. Archit. News 23, 1, 20-24.

Xue, J. And Huang, C.-H. 1997. Reuse-driven tiling for data locality. In Proceedings of the 10th Workshop on Languages and Compilers for Parallel Computing. Springer, Berlin, 16-33.

Yang, X., Zhang, Y., Xue, J., Rogers, I., Li, G., AND Wang, G. 2008. Exploiting loop-dependent stream reuse for stream processors. In Proceedings of the 17th International Conference on Parallel Architectures and Compilation Techniques. ACM, New York, 22-31.

YANG, X. J., YAN, X. B., XING, Z. C., Deng, Y., JiAng, J., AND Zhang, Y. 2007. A 64-bit stream processor architecture for scientific applications. SIGARCH Comput. Archit. News 35, 2, 210-219.

Zhang, Y., Yang, X., Wang, G., Rogers, I., Li, G., Deng, Y., and Yan, X. $2008 . \quad$ Scientific computing applications on a stream processor. In Proceedings of the International Symposium on Performance Analysis of Systems and Software. IEEE, Los Alamitos, CA, 105-114.

Received March 2009; revised October 2009; accepted March 2010 